\title{
Threatened medicinal and economic plants of the Sudan Savanna in Katsina State, northwestern Nigeria
}

\begin{tabular}{|c|c|}
\hline \multicolumn{2}{|c|}{$\begin{array}{l}\text { Authors: } \\
\text { Abubakar Bello }^{1} \\
\text { Sirajo Jamaladdeen }^{1} \\
\text { Muhammad T. Elder }^{1} \\
\text { Samaila S. Yaradua } \\
\text { Sulaiman S. Kankara }^{1} \\
\text { Nasiru H. Wagini }^{1} \\
\text { Charles H. Stirton }^{2} \\
\text { Muthama Muasya }^{2}\end{array}$} \\
\hline $\begin{array}{l}\text { Affiliations: } \\
{ }^{1} \text { Center for Bic } \\
\text { Conservation, } \\
\text { Biology, Umar } \\
\text { University, Kat }\end{array}$ & $\begin{array}{l}\text { diversity and } \\
\text { Department of } \\
\text { ausa Yar'adua } \\
\text { sina, Nigeria }\end{array}$ \\
\hline $\begin{array}{l}{ }^{2} \text { Bolus Herbar } \\
\text { Department o } \\
\text { Sciences, Univ } \\
\text { Town, Cape to }\end{array}$ & $\begin{array}{l}\text { um, } \\
\text { f Biological } \\
\text { ersity of Cape } \\
\text { wn, South Africa }\end{array}$ \\
\hline $\begin{array}{l}\text { Correspondin } \\
\text { Abubakar Bell } \\
\text { abubakar.bell } \\
\text { ac.za }\end{array}$ & $\begin{array}{l}\text { g author: } \\
\text { o, } \\
@ \text { alumni.uct. }\end{array}$ \\
\hline $\begin{array}{l}\text { Dates: } \\
\text { Received: } 28 \\
\text { Accepted: } 07 \\
\text { Published: } 04\end{array}$ & $\begin{array}{l}\text { ct. } 2017 \\
\text { Apt. } 2018 \\
\text { Apr. } 2019\end{array}$ \\
\hline $\begin{array}{l}\text { How to cite th } \\
\text { Bello, A., Jam } \\
\text { Elder, M.T., Ya } \\
\text { Kankara, S.S., } \\
\text { et al., 2019, ' } \\
\text { medicinal and } \\
\text { plants of the } \\
\text { Savanna in Ka } \\
\text { northwestern } \\
\text { Bothalia 49(1) } \\
\text { https://doi.or } \\
\text { v49i1.2325 }\end{array}$ & $\begin{array}{l}\text { is article: } \\
\text { laddeen, S., } \\
\text { radua, S.S., } \\
\text { Wagini, N.H. } \\
\text { hreatened } \\
\text { economic } \\
\text { udan } \\
\text { sina State, } \\
\text { Nigeria', } \\
\text { a2325. } \\
\text { /10.4102/abc. }\end{array}$ \\
\hline $\begin{array}{l}\text { Copyright: } \\
\text { (C) 2019. The A } \\
\text { Licensee: AOS } \\
\text { is licensed unc } \\
\text { Creative Comr } \\
\text { Attribution Lic }\end{array}$ & $\begin{array}{l}\text { uthors. } \\
\text { IS. This work } \\
\text { ler the } \\
\text { nons } \\
\text { ense. }\end{array}$ \\
\hline Read online: & \\
\hline 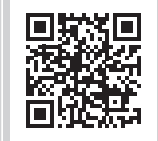 & $\begin{array}{l}\text { Scan this QR } \\
\text { code with your } \\
\text { smart phone or } \\
\text { mobile device } \\
\text { to read online. }\end{array}$ \\
\hline
\end{tabular}

Background: The loss of biodiversity in Nigeria is escalating alarmingly. However, there is generally a paucity of information as to what taxa are endangered because of a dearth of functioning conservation agencies in Nigeria.

Objectives: The aim of this research is to record the endangered medicinal and other economic plant species in the Sudan Savanna vegetation in Katsina and to provide an assessment of the various threats faced by these plants.

Method: Medicinal plants were identified through oral interviews with traditional medical practitioners within the study area. Conservation statuses were assessed using a bespoke data collection and assessment form; the data were then evaluated using the International Union for the Conservation of Nature Red List categories and criteria.

Results: A total of 169 species belonging to 62 families were recorded. Of these, 43 taxa were reported to be used for ethnomedicinal practices. It was found that more than half (108) of the 169 species were threatened with extinction and one taxon (Xeroderris stuhlmannii [Taub.] Mendonca \& Sousa) qualifies as being Extinct locally. Threats recorded include overexploitation $(24 \%)$, agriculture $(15 \%)$, deforestation and desertification $(12 \%$ each), invasive plants $(11 \%)$, urban residential development (7\%) and erosion (6\%).

Conclusion: Most of the plants are already under threat and require urgent conservation measures. The data point to the critical need for further research into conservation strategies and a more sustainable use of threatened plants. We recommend that the Nigerian government should establish a national Red List agency and ensure effective protected area management and community-based natural resources management.

Keywords: conservation; endangered species; Hausa community; IUCN; threat categories; Nigeria; Sudan Savanna.

\section{Introduction}

The loss of biodiversity in Nigeria is escalating alarmingly ('Assessment of the threats to biodiversity' Convention on Biological Diversity [CBD] n.d.; Ihunweze n.d.). This is more pronounced in seven states across the northwestern and northeastern geopolitical zones of Nigeria, which are threatened by the adverse effect of desertification, posing a significant threat to agriculture, food security and water resources (Dike \& Obembe 2012; Gbile 1992; Wakili 2016). These seven states, mostly bordering the Republic of Niger and characterised by Sudano-Sahelian Savanna, are grappling with the challenge of desertification, which advances at $0.6 \mathrm{~km}$ every year, leading to soil erosion and disruption of the ecosystem (Smith 2000: 2). This adverse effect is attributed primarily to persistent deforestation and a loss of ground cover, extensive overgrazing and other faulty farming practices (Borokini 2014; Dike \& Obembe 2012; Emma-Okafor \& Ibeawuchi 2010). The vegetation cover of the Savanna is economically and medicinally important as a large number of Africans use medicinal and aromatic plants that are known to be reservoirs of curative elements in the treatment of various diseases ranging from malaria, diabetes, mental disorders, cancer and hypertension to HIV and AIDS (Bello et al. 2011a, 2011b; Dike \& Obembe 2012; Okigbo 2009). However, increasing demand, coupled with harmful unsustainable collecting habits, threatens the survival of these plants as well as the livelihood of the general population who rely on the overall natural system (Borokini 2014; Emma-Okafor \& Ibeawuchi 2010).

Katsina State lies in the Sudan Savanna vegetation zone (Keay 1949; White 1983) between $12^{\circ} 15^{\prime} \mathrm{N}$ latitudeand $7^{\circ} 30^{\prime}$ Elongitudeand is one of the 11 states that comprise thenorthwestern and northeastern geopolitical zones of Nigeria, which are impacted by the adverse effects of desertification and 
excessive indiscriminate felling of trees for firewood and timber (Bello pers. obs.; Gbile 1992). The plants that are the most threatened are the numerous medicinal and economically important trees, which were once found abundantly in farmlands across the region, but which are no longer easy to come by. Some of these species include Neocarea macrophylla (Sabine) Prance ex F.White (Gawasa), Sclerocarya birrea (A.Rich) Hochst. (Daniya), Detarium microcarpum Harms. (Taura) and Prosopis africana (Guill. \& Perr.) Taub. (Kirya) (Borokini 2014; Emma-Okafor \& Ibeawuchi 2010; Mudansiru et al. 2016). There is therefore a need for a more sustainable forest resource management to ensure that the current generation utilises the available natural resources wisely without compromising the availability of the resources for future generations (Chukwuma, Soladoye \& Feyisola 2015; Taylor 2015).

A major impediment to a more sustainable management is the lack of information on which taxa are endangered due to the paucity of functioning conservation agencies in Nigeria (CBD 2015; 'Red List 2013: Threatened species across the regions of the world'). The major significance of this study is, therefore, to provide up-to-date information about endangered plant species in the Sudan Savanna vegetation zone in Katsina, in accordance with the guidelines set by the International Union for the Conservation of Nature (IUCN). The study also aimed at providing criteria to determine the relative rate of the risk of extinction of medicinal plants, with the main purpose being to catalogue and highlight those plants that are facing a risk of extinction. In addition, we want to assess previous recommendations about conserving the remaining patches of the available plant resources. Specifically, the objectives of this study are to (1) identify the indigenous medicinal and economic plants used by the people of Katsina State; (2) assess the conservation status of the indigenous medicinal and economic plants of the Katsina Sudan Savanna based on the Red Listing criteria developed by the IUCN; and (3) outline the various threat categories affecting the plants.

Internationally, 199 countries have signed an accord to create biodiversity action plans that will aim to protect endangered and other threatened species (Hariramamurthi 2000). One of the recent efforts by the Nigerian government to reduce the loss of biodiversity was the signing of the bill by the president of Nigeria on 30 December 2016 to stop the trafficking of endangered species (Wakili 2016). In Katsina State, the Ministry of Environment implemented the Great Green Wall Project (greatgreenwall.org; UN Convention to Combat Desertification), which seeks to establish shelter belts in dry regions of the world as a step to tackle the menace of desertification. To the best of our knowledge, there has never been an up-to-date assessment of the status and distribution of threatened species in Katsina as has been reported from some other places, for example the southern part of Nigeria (Chukwuma et al. 2015; Dike \& Obembe 2012; Emma-Okafor \& Ibeawuchi 2010), Senegal (Zizka et al. 2015) and southern Africa (Victor \& Dold 2003). The list compiled here is primarily to identify those taxa most in need of conservation attention, thereby assisting conservationists, farmers, landowners and land managers by providing clear information for conservation of threatened species.

\section{Methodology Study area}

This research was carried out in the Sudan Savanna vegetation zone in Katsina State, northwestern Nigeria (Keay 1949; White 1983). The area consists of nine local government areas including Jibia, Katsina, Kaita, Mashi, Maiadua, Daura, Sandamu, Zango and Baure (Figure 1). The area has a mean annual rainfall of about $800 \mathrm{~mm}-1000 \mathrm{~mm}$ (Olofin 1985). The vegetation is characterised by a variety of scattered trees in an expanse of grassland (Olofin 1985). The area is known to enjoy four distinct seasons: a dry and cool season, which starts from around November and ends in late March and is characterised by Harmattan winds; a dry and hot season, which is a short transitional period from March to about midMay characterised by the warmest temperature of the year; a wet and warm season, which starts around May and ends in October during which $90 \%$ of the annual rainfall is received; and lastly, a dry and warm season, which starts from the end of October to mid-November (Olofin 1985).

\section{Users consulted in the study and sampling techniques}

To determine which medicinal and economic important plants were being used in the region, we targeted users such as herbalists, traditional medical practitioners, traditional midwives, housewives, farmers and other elders who have practised with or used medicinal plants. These groups effectively sub-sampled the larger population thereby enabling us to generalise the findings more easily. These targeted consultees were identified after conferring with village heads in the study area. The number of consultees varied according to the size of the population of the study area. This ensured a proportionate representation of the share of the population. Random sampling was adopted to select villages in the north, east, south and west of each of the nine local government units in the study area thereby minimising bias and oversimplification of the results.

\section{Sources of information on medicinal plants and Red List data}

The primary source of data was derived from (1) the questionnaire designed to obtain information about plants used for medicinal purposes (Appendix 1) and (2) the species collection or assessment form for conservation assessment (Appendix 2).

The secondary source of data includes the IUCN Red List website (http://www.iucnredlist.org), which was used to ascertain the global conservation status of each species; GeoCAT (http://geocat.kew.org/) and Global Biodiversity Information Facility (GBIF) (http://www.gbif.org/). The IUCN Red List is the world's most comprehensive information source on the extinction risk of plant and animal species (Rodrigues et al. 2006). The information in the IUCN Red List is widely used to inform biodiversity conservation policy and practice, making the IUCN Red List a preferable method 


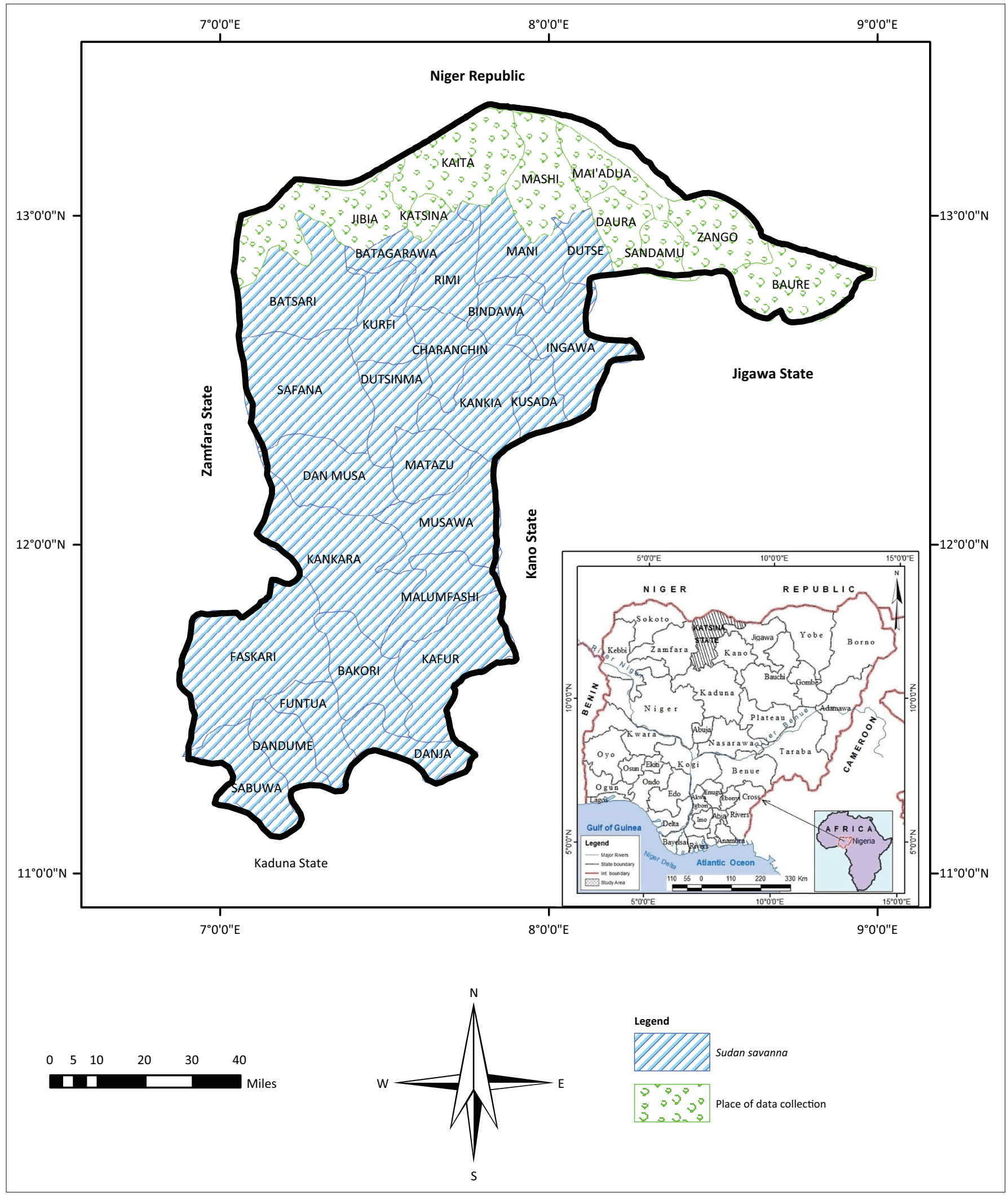

FIGURE 1: Map of Katsina State, northwestern Nigeria, showing the study area.

for conservation decision-making (see MedPlant 2015). GeoCAT performs rapid geospatial analysis for Red Listing taxa. It utilises spatially referenced primary occurrence data and focuses on two aspects of the geographic range of a taxon: the extent of occurrence (EOO) and the area of occupancy (AOO), which form part of the IUCN Red List categories and criteria and have often proved challenging to obtain in an accurate, consistent and repeatable way (Bachman et al. 2011). GBIF is an open-data research infrastructure funded by all the governments in the world and aimed at providing anyone, anywhere access to data about all types of life on the Earth. 


\section{Data collection}

Field study trips in the study area were made between June 2015 and June 2017. Plants of medicinal and economic importance were identified through oral interviews with the targeted groups representing the categories mentioned above. On the advice of these informants, plants were shortlisted for inclusion in the study. Then, the researchers, with the help of the local individuals, proceeded to the field to make conservation assessments of the listed plants using the pro forma noted in the previous section (Appendix 2). Each of the listed species was assessed individually in the field. Additionally, the informants were asked various questions regarding the conservation status of the medicinal plants. These helped in outlining the major threats causing the reduction in population size of the various medicinal and economic plants listed. To ensure that only valid samples and information are used in the study, we used both semi-structured and unstructured interviews as the best approach because in a rural or indigenous context, people often do not respond well to highly structured interview approaches. We went to the interview with a few questions relating to the topic of discussion. One of the advantages of this is that if the conversation deviates significantly, the interviewers can gently steer it back to their topics of focus. We ensured that our interviewees were at ease by starting the interview with informal chats. We also explained how the data would be used. We allowed the interviewees to do most of the talking without interruption and used simple, clear language and short questions. The technique for eliciting information during informal conversations is to get people on to a topic (see MedPlant 2015).

Information on the decline in the population size of the species, decline in the $\mathrm{AOO}, \mathrm{EOO}$, loss of habitat, actual or potential level of exploitation and effects of introduced taxa noted in the field were used in the evaluation of the taxa following the IUCN Red List criteria version 3.1 (IUCN 2012a, 2012b: Appendix 3). Voucher collections of the taxa facing any of the three categories of threat were made and deposited in the herbarium of Umaru Musa Yarádua University, Katsina (UMYUK).

\section{Data analysis}

The IUCN Red List criteria were used to classify species according to one of the three categories of threat, or a category of lower risk (IUCN 2001; SANBI 2010, see also Appendix 3). Briefly, the three categories of threat in order of decreasing risk of extinction were Critically Endangered (CR), Endangered (EN) and Vulnerable (VU). If a taxon no longer exists in the wild, it is classified as Extinct (EX) or Extinct in the Wild (EXW). Categories of lower risk are Near Threatened (NT) or Least Concern (LC). The NT category has been used where there is a concern that the taxon may become threatened in the future, but where the taxon does not qualify as 'threatened with extinction'. Taxa that were not threatened with extinction are listed as LC. The minimum amount of information required for performing an assessment is the total distribution area of the taxon (EOO), the number of locations in which it grows $(\mathrm{AOO})$ and whether or not it is declining (the reason is also recorded). The $\mathrm{AOO}$ and the EOO were calculated using the GeoCAT tool (Bachman et al. 2011). Where a continuing decline was observed to be affecting the population as a whole, the B criterion was applied; and in some cases, if a percentage decline could be estimated, the A criterion was used. Quite often, for a very narrowly restricted taxon under threat from a stochastic event because of its rarity, the D criterion was used.

\section{Result}

The result of the socio-demographic information of the respondents interviewed during the ethnobotanical survey of the medicinal plants in the study area is shown in Table 1 . The table presented the sex, age and educational status, occupation of the respondents as well as their frequency and percentage. It shows that there are more male respondents, 83 (78.9\%) than female, $22(21.2 \%)$ respondents and that the majority, 33 (31.4\%) falls within the age range of 51-60 years. It also showed that the majority of the respondents, $59(56 \%)$ have no formal education and very few, $5(4.8 \%)$ have tertiary education. In terms of occupation, the majority of the respondents, $42(30.9 \%)$ are traditional medical practitioners (Table 1$)$.

Table 2 shows the list of 43 plant species distributed across 20 families used for the treatment of various common illnesses and ethnomedicinal practices by the people of Katsina. All taxa are listed in alphabetical order by families. Each plant is reported with the type of disease it cures, part of the plant used and the method of usage. The local (Hausa) and scientific names were also provided for the benefit of the native Hausa speakers and the general scientific community, respectively. Fabaceae has the highest record of 15 species, followed by Anacardiaceae (4), Moraceae and Combretaceae with three species each and Connaraceae and Malvaceae with two species each. The remaining families were represented by a single species (Table 2).

Table 3 shows the list of 61 assessed medicinal and economic plants that either may become threatened with

TABLE 1: Socio-demographic information about the respondents $(N=105)$.

\begin{tabular}{lcc}
\hline Bio-data & Frequency $(\boldsymbol{n})$ & Percentage (\%) \\
\hline Sex & & \\
Male & 83 & 78.85 \\
Female & 22 & 21.15 \\
Age & & \\
$20-30$ & 7 & 6.66 \\
$31-40$ & 13 & 12.38 \\
$41-50$ & 22 & 20.95 \\
$51-60$ & 33 & 31.42 \\
$61-70$ & 19 & 18.09 \\
70 and above & 11 & 10.14 \\
Educational status & & \\
Tertiary & 5 & 4.76 \\
Secondary & 14 & 13.33 \\
Primary & 27 & 25.72 \\
No formal education & 59 & 56.19 \\
Occupation & & \\
Farmers & 6 & 5.71 \\
Herbalist & 22 & 20.95 \\
Traditional medical practitioners & 42 & 30.48 \\
Traditional midwives & 22 & 20.95 \\
Housewives & 16 & 15.24 \\
Others & 7 & 6.67 \\
\hline
\end{tabular}

Note: The table presented the sex, age, educational status and occupation of the respondents as well as their frequency and percentage. 
TABLE 2: Medicinal plants used by the people of Katsina.

\begin{tabular}{|c|c|c|c|c|c|}
\hline Scientific name & Family & Local name & Part use & Disease cured & Method of preparation and administration \\
\hline Adansonia digitata $\mathrm{L}$. & Malvaceae & Kuka & L & Piles & Powder of leaves is taken with soup. \\
\hline Allium sativum $\mathrm{L}$. & Amaryllidaceae & Tafarnuwa & B & Colds & Powder of bulb taken orally. \\
\hline Albizia chevalieri Harms. & Fabaceae & Katsari & B & Stomach ache & Soaked in water, the extract is taken orally. \\
\hline Anogeissus leiocarpus (DC.) (Guill. And Perr.) & Combretaceae & Marke & B & Piles and fever & $\begin{array}{l}\text { Soaked in water, a cup full of the extract is taken } \\
\text { daily. }\end{array}$ \\
\hline Balanites aegyptiaca (L.) Delile & Zygophyllaceae & Aduwa & $\mathrm{F}$ & Piles & Fruit is taken orally. \\
\hline Bauhinia rufescens Lam. & Fabaceae & Tsattsagi & B & Dysentery & Soaked in water and taken orally. \\
\hline Byrsocarpus coccineus Schum. and Thonn. & Connaraceae & Tsamiyar kasa & WP & Supplementing breast milk & Powder taken with milk or pap. \\
\hline Zaleya pentandra (L.) C.Jeffrey & Nyctaginaceae & Gadon Maciji & $\mathrm{T}$ & Stomach ache & An infusion of root and leaves is taken orally. \\
\hline Boswellia dalzielii Hutch. & Burseraceae & Hano & $\mathrm{T}$ & Piles & $\begin{array}{l}\text { Soaked in water for } 3 \text { days and the extract then } \\
\text { taken orally. } \\
\text { Root is mixed with honey. }\end{array}$ \\
\hline Calotropis procera (Aiton) Dryand. & Asclepiadaceae & Tunfafiya & $\mathrm{T}$ & Cancer, eye problems & $\begin{array}{l}\text { Powder smeared on the cancerous area. Fruit } \\
\text { liquid is used as an eye drop. }\end{array}$ \\
\hline Chamaecrista mimosoides (L.) Greene & Fabaceae & Bagaruwar kasa & $\mathrm{L}$ & Skin rashes & Ointment is rubbed onto the skin. \\
\hline Combretum micranthum G.Don & Combretaceae & Geza & L & General well being & Decoction is taken orally. \\
\hline Rourea coccinea (Schum. \& Thonn.) Benth. & Connaraceae & Tsamiyar kasa & $\mathrm{F}$ & Piles & Fruit soaked in water and then taken orally. \\
\hline Citrus aurantiifolia (Christm.) Swingle & Rutaceae & Lemun Tsami & $\mathrm{F}$ & Fever and mouth rashes & $\begin{array}{l}\text { Steaming using the decoction. Fruit juice is } \\
\text { applied to mouth. }\end{array}$ \\
\hline Detarium microcarpum Harms. & Fabaceae & Taura & $B, F$ & Piles & Fruit or decoction is taken orally. \\
\hline Diospyros mespiliformis Hochst. ex A.DC. & Ebenaceae & Kanya & B & Piles & Soaked in water and taken orally. \\
\hline Entada africana Guill. \& Perr. & Fabaceae & Tawatsa & B & Stomach ache & Cup full of decoction is taken orally. \\
\hline Erythrina senegalensis DC. & Fabaceae & Minjirya & B & Dysentery & Powder is taken orally with pap. \\
\hline Faidherbia albida (Delile) A.Chev. & Fabaceae & Gawo & B & Body pain & Soaked then taken orally. \\
\hline Ficus trichopoda Baker & Moraceae & Baure & B & Diarrhoea & The decoction is taken orally. \\
\hline Ficus thonningii Blume & Moraceae & Cediya & B & Fever & $\begin{array}{l}\text { Powder is added on a pap or soaked in water } \\
\text { then taken orally. }\end{array}$ \\
\hline Ficus platyphylla Delile & Moraceae & Gamji & B & Piles & Soaked and taken orally. \\
\hline Guiera senegalensis J.F.Gmel. & Combretaceae & Sabara & L & Diarrhoea and vomiting & Powder of leaves is taken orally. \\
\hline Indigofera astragalina DC. & Fabaceae & $\begin{array}{l}\text { Kai kai koma kan } \\
\text { mashekiya }\end{array}$ & WP & Arthritis & Powder applied to affected area. \\
\hline Jatropha curcas L. & Euphorbiaceae & Cindazugu & WP & Body pains & The decoction is taken orally. \\
\hline $\begin{array}{l}\text { Leptadenia lancifolia } \\
\text { (Schumach. \& Thonn) Decne. }\end{array}$ & Apocynaceae & Yadiya & L & Ulcers & Cooked leaves are taken as food. \\
\hline Mangifera indica $\mathrm{L}$. & Anacardiaceae & Mangoro & B & Malaria & The boiled decoction is taken orally. \\
\hline Moringa oleifera Lam. & Moringaceae & Zogala & L & $\begin{array}{l}\text { Blood tonic and general well } \\
\text { being }\end{array}$ & Cooked and taken as food. \\
\hline Parkia biglobosa (Jacq.) G.Don & Fabaceae & Dorawa & B & Dysentery & The decoction is taken orally. \\
\hline Psidium guajava $\mathrm{L}$. & Myrtaceae & Gwaba & L & Fever & The decoction is orally taken for 3 days. \\
\hline Bauhinia reticulata DC. & Fabaceae & Kalgo & $\mathrm{R}$ & Piles & Root extract plus red potash is taken orally. \\
\hline $\begin{array}{l}\text { Ozoroa mucronata (Bernh.) } \\
\text { R.Fern. \& A.Fern. }\end{array}$ & Anacardiaceae & Kasheshe & L & Vomiting & Leaf powder added to milk and taken orally. \\
\hline Prosopis africana (Guill. \& Perr.) Taub. & Fabaceae & Kirya & B & Piles & Powdered bark applied to the affected area. \\
\hline Sclerocarya birrea (A.Rich.) Hochst. & Anacardiaceae & Danya & B & Body pain & Stem bark soaked in water and taken orally. \\
\hline Senna occidentalis (L.) Link & Fabaceae & Tafasar masar & L & Fever & The decoction is taken orally. \\
\hline Strychnos spinosa Lam. & Loganiaceae & Kokiya & B & Ulcers & Soaked and taken orally. \\
\hline Tamarindus indica $\mathrm{L}$. & Fabaceae & Tsamiya & $\mathrm{F}$ & Stomach upset & Soaked in water and taken orally. \\
\hline Vachellia nilotica (L.) P.J.H.Hurter \& Mabb. & Fabaceae & Bagaruwa & $\mathrm{F}$ & Healing piles and wounds & $\begin{array}{l}\text { A decoction is taken with pap every morning for } \\
\text { at least a week. It is also soaked and tied to } \\
\text { wounds for healing. }\end{array}$ \\
\hline Vachellia sieberiana (DC.) Kyal. \& Boatwr. & Fabaceae & Farar kaya & WP & Easing joint pain & Soaked in water and taken orally. \\
\hline Ziziphus jujuba Mill. & Rhamnaceae & Magarya & L & Blood clotting & Leaf powder is applied to wound. \\
\hline
\end{tabular}

Note: The table lists 43 plant species from 20 plant families used in the treatment of various common illness and ethnomedicinal practices.

$B$, bark; F, fruit; L, leaves; $R$, root; $T$, tuber; $W P$, whole plant.

extinction in the future, or for which there is some conservation concern, that is, the NT category (36), or those that are not threatened with any extinction risk and did not qualify to be listed in the NT category, that is, the LC category (25). Table 4 showed the list of 108 taxa that are either EX or are threatened with extinction. Of these, one taxon qualifies as EX, 36 as CR, 25 as EN and 46 as VU (see also Figure 2).
Figure 2 shows the percentage of each of the threat categories affecting the various medicinal and economic plants in the study area. Fifteen per cent are LC, 21\% NT, 27\% VU, 15\% $\mathrm{EN}, 21 \% \mathrm{CR}$ and $1 \% \mathrm{EX}$. The various categories of threats faced by the plants and the number of taxa affected in each of the threat categories are shown in Figure 3. These are (in descending order): overexploitation 26 (24.1\%); agricultural practices 16 (14.8\%); deforestation and desertification 13 
TABLE 3: Medicinal and economic plant taxa that either may become threatened with extinction in the future, or for which there is some conservation concern, that is, the Near Threatened category (36), or those that are not threatened with any extinction risk and did not qualify to be listed in the Near Threatened category, that is, the Least Concern category (25). These taxa (61) do not meet the criteria for being listed as 'threatened with extinction' according to the International Union for the Conservation of Nature version 3.1.

\begin{tabular}{|c|c|c|c|c|}
\hline Family & Species & Local name & Local assessment & Global assessment \\
\hline Aizoaceae & Zaleya pentandra (L.) C.Jeffrey & Gadon maciji & NT & $\mathrm{NE}$ \\
\hline Amaranthaceae & Amaranthus spinosus L. & Zarangade & NT & NE \\
\hline Amaryllidaceae & Allium sativum $\mathrm{L}$. & Tafarnuwa & NT & NE \\
\hline Amaryllidaceae & Scadoxus multiflorus (Martyn) Raf. & Albasar kwadi & NT & NE \\
\hline Anacardiaceae & Mangifera indica $\mathrm{L}$. & Mangwaro & NT & DD \\
\hline Anacardiaceae & Ozoroa mucronata (Bernh.) R.Fern \& A.Fern. & Kasheshe & LC & NE \\
\hline Anarcadiaceae & Anacardium occidentale L. & Yazawa & LC & NE \\
\hline Apocynaceae & Calotropis procera (Aiton) Dryand. & Tumfafiya & NT & $\mathrm{NE}$ \\
\hline Apocynaceae & Leptadenia lancifolia (Schumach. \& Thonn) Decne. & Yadiya & NT & $\mathrm{NE}$ \\
\hline Arecaceae & Borassus aethiopum Mart. & Giginya & NT & NE \\
\hline Asteraceae & Vernoniastrum ambiguum (Kotschy \& Peyr.) H.Rob. & Tattaba & LC & $\mathrm{NE}$ \\
\hline Brassicaceae & Lepidium sativum $\mathrm{L}$. & Zamantarori & LC & NE \\
\hline Caricaceae & Carica papaya L. & Gwanda & LC & DD \\
\hline Cleomaceae & Cleome gynandra L. & Gasaya & NT & NE \\
\hline Commelinaceae & Commelina caroliniana Walter. & Balasana & NT & NE \\
\hline Convolvulaceae & Evolvulus alsinoides (L.) L. & Kafi malam & NT & $\mathrm{NE}$ \\
\hline Convolvulaceae & Ipomoea asarifolia (Desr.) Roem. \& Schult. & Duman kada & NT & NE \\
\hline Cucurbitaceae & Citrullus lanatus (Thunb.) Matsum. \& Naka. & Guna & NT & NE \\
\hline Cyperaceae & Cyperus articulatus L. & Kajiji & NT & NE \\
\hline Cyperaceae & Cyperus rotundus L. & Aya Aya & NT & LC \\
\hline Euphorbiaceae & Chrozophora senegalensis (Lam.) A.Juss ex Spreng. & Damaigi & NT & NE \\
\hline Euphorbiaceae & Euphorbia balsamifera Aiton & Aliyara & NT & NE \\
\hline Euphorbiaceae & Jatropha curcas L. & Cin da zugu & NT & NE \\
\hline Euphorbiaceae & Euphorbia convolvuloides Hoschst. ex Benth. & Nonon kurciya & NT & NE \\
\hline Fabaceae & Senegalia polyacantha (Willd.) Seigler \& Ebinger & Karaki & NT & $\mathrm{NE}$ \\
\hline Fabaceae & Vachellia sieberiana (DC.) Kyal. \& Boatwr. & Farar kaya & LC & $\mathrm{NE}$ \\
\hline Fabaceae & Bauhinia reticulata DC. & Kalgo & NT & NE \\
\hline Fabaceae & Crotalaria juncea $\mathrm{L}$. & Bakar biyar rana & LC & $\mathrm{NE}$ \\
\hline Fabaceae & Indigofera astragalina DC. & Kaikai koma kan mashekiya & NT & NE \\
\hline Fabaceae & Senna obtusifolia (L.) H.S.Irwin \& Barneby & Tafasa & LC & $\mathrm{NE}$ \\
\hline Fabaceae & Senna occidentalis (L.) Link & Tafasar Masar & LC & NE \\
\hline Fabaceae & Senna singueana (Delile) Lock & Runhu & NT & $\mathrm{NE}$ \\
\hline Lamiaceae & Leucas martinicensis (Jacq.) R.Br. & Bunsurun fadama & LC & NE \\
\hline Lamiaceae & Ocimum basilicum $\mathrm{L}$ & Daddoya & LC & $\mathrm{NE}$ \\
\hline Malvaceae & Gossypium hirsutum L. & Gurya & LC & NE \\
\hline Malvaceae & Hibiscus sabdariffa L. & Sobo & LC & $\mathrm{NE}$ \\
\hline Malvaceae & Sida ovata Forssk. & Miyar tsanya & LC & NE \\
\hline Moringaceae & Moringa oleifera Lam. & Zogala & NT & $\mathrm{NE}$ \\
\hline Myrtaceae & Psidium guajava $\mathrm{L}$. & Gwaba & NT & NE \\
\hline Nyctaginaceae & Boerhavia diffusa $\mathrm{L}$. & Babba jibji & NT & NE \\
\hline Pedaliaceae & Ceratotheca sesamoides Endl. & Yaudo & LC & NE \\
\hline Pedaliaceae & Sesamum alatum Thonn. & Ridin barewa & LC & $\mathrm{NE}$ \\
\hline Poaceae & Andropogon gayanus Knuth & Gamba & NT & NE \\
\hline Poaceae & Cynodon dactylon (L.) Pers. & Tsarkiyar zomo & LC & $\mathrm{NE}$ \\
\hline Poaceae & Pennisetum glaucum (L.) R.Br. & Gero & LC & NE \\
\hline Poaceae & Pennisetum hordeoides (Lam.) Steud. & Kyasuwa & LC & $\mathrm{NE}$ \\
\hline Poaceae & Phragmites australis (Cav.) Trin. ex Steud. & Katala & LC & LC \\
\hline Poaceae & Cenchrus biflorus Roxb. & Karangiya & NT & NE \\
\hline Poaceae & Digitaria debilis (Desf.) Wild. & Harkiya & NT & NE \\
\hline Poaceae & Eragrostis tremula Hochst. ex Steud. & Burburuwa & NT & NE \\
\hline Rubiaceae & Mitragyna inermis (Willd.) Kuntze & Giyayya & LC & NE \\
\hline Rubiaceae & Mitracarpus hirtus (L.) DC. & Gogamasu & NT & NE \\
\hline Rubiceae & Spermacoce stachydea DC. & Alkamar Turwa & NT & NE \\
\hline Rutaceae & Citrus aurantiifolia (Christm.) Swingle & Lemun tsami & LC & NE \\
\hline Solanaceae & Schwenkia americana Kunth & Dandana & NT & NE \\
\hline Solanaceae & Solanum americanum Mill. & Gautan kaji & LC & NE \\
\hline Vitaceae & Cissus populnea Guill. \& Perr. & Loda & LC & NE \\
\hline
\end{tabular}

NE, No entry, DD, Data deficient; NT, Near Threatened; LC; Least Concern. 
TABLE 4: Taxa threatened with local extinction in Katsina, assessed according to the International Union for the Conservation of Nature categories and criteria version 3.1.

\begin{tabular}{|c|c|c|c|c|}
\hline Family & Species & Local name & Local assessment & Global assessment \\
\hline Acanthaceae & Hygrophila auriculata Schumach. & Zazargiwa & VU B1ab (i,ii);D2 & LC \\
\hline Acanthaceae & Peristrophe bicalyculata (Retz) Nees & Tubanin dawaki & VU A1 c,d & NE \\
\hline Aizoaceae & Tricanthema portulacastrum L. & Dankalin yara & VU B1ab(v);D2 & NE \\
\hline Amaryllidaceae & Urelytrum giganteum Pilg. & Jema & CR B1ab(iv,v);D1 & NE \\
\hline Anacardiaceae & Lannea acida A.Rich & Faru & VU B1abc (i,ii,iii,iv,v);D2 & NE \\
\hline Anacardiaceae & Sclerocarya birrea (A.Rich.) Hochst. & Danya & EN C2 b, D\&E & NE \\
\hline Annonaceae & Annona senegalensis Pers. & Gwandar daji & EN Aide; C2a(i) & NE \\
\hline Apocynaceae & Caralluma dalzielii N.E.Br. & Karan masallaci & EN B1ab (i,ii,v) & NE \\
\hline Apocynaceae & Pergularia tomentosa $\mathrm{L}$. & Fatakka & VU B2ab(iv,v);D2 & NE \\
\hline Apocynceae & Adenium obesum (Forssk.) Roem. \& Schult. & Karya & EN D & NE \\
\hline Araceae & Anchomanes difformis (Blume) Engl. & Hantsar gada & VU D2 & NE \\
\hline Araceae & Stylochiton lancifolius Kolchy \& Peyr. & Kunnen Jakki & VU B2ab(iv,v);D2 & NE \\
\hline Arecaceae & Phoenix dactylifera $\mathrm{L}$. & Dabino & VU B1ab(i,ii,iv,v);D2 & NE \\
\hline Arecaceae & Hyphaene thebaica (L.) Mart. & Goruba & VU D2 & LC \\
\hline Asteraceae & Acanthospermum hispidum DC. & Yawo & VUA1 a,b & NE \\
\hline Asteraceae & Artemisia annua $\mathrm{L}$. & Tazargade & VU & NE \\
\hline Asteraceae & Baccharoides adoensis (Sch. Bip. ex Walp.) H.Rob. & Dumashi & VUA2 c,d & NE \\
\hline Asteraceae & Centaurea acarnanica (Matthäs) Greuter & Dayi & VUA2 c,d & NE \\
\hline Asteraceae & Centaurea perrottetii DC. & Surandi & VU B1ab (i,ii,iv,v) & NE \\
\hline Asteraceae & Vernonia amygdalina Delile & Shuwaka & EN B1 b(iii) & NE \\
\hline Asteraceae & Vernonia kotschyana (Sch.Bip. ex Walp.) & Kumbura fage & VU B1ab(iv,v);D2 & NE \\
\hline Bignoniaceae & Stereospermum kunthianum Cham. & Sansami & VUA2 c,d & NE \\
\hline Bombacaceae & Bombax brevicuspe Sprague & Kurya & $C R A 1 b, B 2 a \& C 2 b$ & VU A1cd \\
\hline Boraginaceae & Cordia africana Lam. & Alilliba & CR B12b (i,ii,iii,iv,v) & NE \\
\hline Brassicaceae & Lepidium sativum L. & Zamantarori & VU B1ab(iv,v);D2 & NE \\
\hline Burseraceae & Boswellia dalzielii Hutch. & Hano & CR B1ab (i,ii,iii) + 2ab (i,ii,iii,iv) & NE \\
\hline Burseraceae & Commiphora kerstingii Engl. & Baazana & ENA2 d,e \&E & NE \\
\hline Burseraceae & Commiphora hildebrandtii (Engl.) Engl. & Dashi & EN B1ab (i,ii,iii); 2ac(ii) & NE \\
\hline Capparaceae & Cadaba farinosa Forssk. & Bagai & CR B1a + 2ab (ii,iv,v) & NE \\
\hline Capparaceae & Crateva adansonii DC. & Ungududu & CR B12b (i,ii,iii,iv,v) & NE \\
\hline Chrysobalanaceae & Neocarya macrophylla (Sabine) Prance ex F.White & Gawasa & EN B1ab(i,ii,iv,v) & NE \\
\hline Cochlospermaceae & Cochlospermum tinctorium Perr. ex A.Rich. & Rawaya & EN B1ab (i,iii,iv,v); 2ab(i,ii,iii,iv,v) & NE \\
\hline Combretaceae & Anogeissus leiocarpa (DC.) Guill. \& Perr. & Marke & EN C2b; D. & NE \\
\hline Combretaceae & Combretum glutinosum Perr. ex DC. & Tarauniya & CR B2ab (i,ii,v) & NE \\
\hline Combretaceae & Combretum micranthum G.Don & Geza & VU D2 & NE \\
\hline Combretaceae & Combretum molle R.Br. ex G.Don & Gogen Damo & VU B1ab (i,iii,v); D2 & NE \\
\hline Combretaceae & Guiera senegalensis J.F.Gmel. & Sabara & VUA1 d,e & NE \\
\hline Combretaceae & Terminalia avicennioides Guill. \& Perr. & Baushe & CR B1ab (iv, v); D1 & NE \\
\hline Connaraceae & Rourea coccinea (Schumach. \& Thonn.) Benth. & Tsamiyar kasa & VUB2 b(i,ii,iii) & NE \\
\hline Cucurbitaceae & Momordica balsamina $\mathrm{L}$. & Garahuni & VU A1abcde;D2 & NE \\
\hline Dioscoreaceae & Dioscorea bulbifera $\mathrm{L}$. & Tuwon biri & EN B1ab (iii,iv,v) & NE \\
\hline Ebenaceae & Diospyros mespiliformis Hochst. ex A.DC. & Kanya & $C R B 1 b(i) \& C 2 b$ & NE \\
\hline Euphorbiaceae & Chrozophora senegalensis (Lam.) A.Juss. ex Spreng. & Damaigi & VU B1ab (i,ii,iii,iv,v) & NE \\
\hline Euphorbiaceae & Euphorbia poissonii Pax. & Tunya & CR D1 & NE \\
\hline Fabaceae & Ptercarpus erinaceus Poir. & Madobiya & VUA1 b,e & NE \\
\hline Fabaceae & Senegalia ataxacantha (DC.) Kyalangaliwa \& Boatwr. & Sarkakiya & VU D2 & NE \\
\hline Fabaceae & Vachellia nilotica (L.) P.J.Hurter \& Mabb. & Bagaruwa & EN A1cde & NE \\
\hline Fabaceae & Senegalia senegal (L.) Britton & Dakwara & VUA2 c,d & NE \\
\hline Fabaceae & Albizia chevalieri Harms. & Katsari & VU D2 & NE \\
\hline Fabaceae & Bauhinia rufescens Lam. & Tsatstsagi & EN A2cde & NE \\
\hline Fabaceae & Cassia arereh Delile & Malga & VU C2a(i) & NE \\
\hline Fabaceae & Chamaecrista mimosoides (L.) Greene & Bagaruwar kasa & EN B2ab (i,ii,iv,v) & NE \\
\hline Fabaceae & Detarium microcarpum Harms. & Taura & CR B1ab (i,ii,iii,iv,v)+2ab (i,ii,iii) & LC \\
\hline Fabaceae & Entada africana Guill. \& Perr. & Tawatsa & CR B1ab(iv,v);D1 & NE \\
\hline Fabaceae & Erythrina senegalensis DC. & Minjirya & $\mathrm{CR} A 2 \mathrm{c} \& \mathrm{~B} 1 \mathrm{~b}(\mathrm{v})$ & LC \\
\hline Fabaceae & Faidherbia albida (Delile) A.Chev. & Gawo & VUA2 c,d & NE \\
\hline Fabaceae & Xeroderris stuhlmannii (Taub.) Mendonca \& Sousa & Jina jina & EX & NE \\
\hline
\end{tabular}


TABLE 4 (Continues...): Taxa threatened with local extinction in Katsina, assessed according to the International Union for the Conservation of Nature categories and criteria version 3.1.

\begin{tabular}{|c|c|c|c|c|}
\hline Family & Species & Local name & Local assessment & Global assessment \\
\hline Fabaceae & Prosopis africana (Guill \& Perr.) Taub. & Kirya & CR B1ab(iv,v);D1 & $\mathrm{NE}$ \\
\hline Fabaceae & Sesbania dalzielii E.Phillips \& Hutch. & Kalumbo & CR A2acd;B1ab(iii) & $\mathrm{NE}$ \\
\hline Fabaceae & Stylosanthes erecta P.Beauv. & Fasafako & VU D2 & $\mathrm{NE}$ \\
\hline Fabaceae & Tamarindus indica $\mathrm{L}$. & Tsamiya & ENA2 b,c \& B2 c(i) & NE \\
\hline Fabaceae & Isoberlinia doka Craib \& Stapf & Doka & VU B1ab(i,iv,v);D2 & LC \\
\hline Fabaceae & Burkea africana Hook. & Bakin makarfo & CR B1ab (iv,v);D1 & $\mathrm{NE}$ \\
\hline Guttiferae & Harungana madagascariensis Lam. ex Poir. & Alillibar rafi & CR B1ab(iv,v);D1 & $\mathrm{NE}$ \\
\hline Lamiaceae & Vitex doniana Sweet & Dinya & VU D2 & NE \\
\hline Loganiaceae & Strychnos spinosa Lam. & Kokiya & EN B2ab(i,ii,iv,v);D1 & $\mathrm{NE}$ \\
\hline Lythraceae & Lawsonia inermis $\mathrm{L}$. & Lalle & EN B1ab(iv,v); D2 & $\mathrm{NE}$ \\
\hline Malvaceae & Adansonia digitata $\mathrm{L}$. & Kuka & VU D2 & $\mathrm{NE}$ \\
\hline Malvaceae & Ceiba pentandra (L.) Gaertn. & Rimi & CRA1 d \& B1 a,b(i,v) D,E & NE \\
\hline Malvaceae & Gossypium barbadense L. & Kada 'yarkarfi & VU B1ab (i,ii,iv,v);D2 & $\mathrm{NE}$ \\
\hline Malvaceae & Pavonia hirsuta Cav. & Zamarke & EN A2acde;B1ab(iv,v) & NE \\
\hline Malvaceae & Pavonia senegalensis (Cav.) Leistner & Tsu & CR A2abcd;C1+2a(i,ii) & $\mathrm{NE}$ \\
\hline Malvaceae & Sterculia setigera Delile & Kukkuki & CR B1ab (iv,v);D1 & $\mathrm{NE}$ \\
\hline Malvaceae & Grewia mollis Juss. & Dargaza & VU B1ab(v);D2 & $\mathrm{NE}$ \\
\hline Meliaceae & Khaya senegalensis (Desv.) A.Juss. & Madacci & VU D2 & VU A1cd \\
\hline Moraceae & Ficus trichopoda Baker & Baure & CR A3 b \&B1(v) & $\mathrm{NE}$ \\
\hline Moraceae & Ficus ingens (Miq.) Miq. & Kawuri & CR A3 b \&B1(v) & $\mathrm{NE}$ \\
\hline Moraceae & Ficus platyphylla Delile & Gamji & ENA2 a & $\mathrm{NE}$ \\
\hline Moraceae & Ficus sur Forssk. & Uwar yara & CR A3b & NE \\
\hline Moraceae & Ficus umbellata Vahl. & Yandi & CR A3b & $\mathrm{NE}$ \\
\hline Moraceae & Ficus vallis-choudae Delile & Lubiya & CR B2ab (i, v);D1 & NE \\
\hline Moraceae & Ficus thonningii Blume & Cediya & VUA3 d & $\mathrm{NE}$ \\
\hline Nymphaeaceae & Nymphaea lotus L. & Bado & VU B2ab(i,ii,iv,v);D2 & NE \\
\hline Olacaceae & Ximenia americana $\mathrm{L}$. & Tsada & CR B1ab(i,ii,iv,v);D1 & $\mathrm{NE}$ \\
\hline Onagraceae & Ludwigia octovalvis (Jacq.) P.H.Raven & Shashatau & CR B1ab (i,ii,iii)+2ab (i,ii,iii) & NE \\
\hline Opiliaceae & Opilia amentacea Roxb. & Rugaggada & EN A3c;B1ab(i,ii,iii,iv) & $\mathrm{NE}$ \\
\hline Orobanchaceae & Striga hermonthica (Delile) Benth. & Gogai & VU B2ab(i,ii,v);D2 & $\mathrm{NE}$ \\
\hline Papaveraceae & Argemone mexicana $\mathrm{L}$. & Kankamarka ta bika & VU D2 & $\mathrm{NE}$ \\
\hline Phyllanthaceae & Bridelia ferruginea Benth. & Kirni & $\mathrm{CR} A 2 \mathrm{~b}, \mathrm{c}, \mathrm{d}, \mathrm{e} \& \mathrm{~B} 1 \mathrm{a}$ & NE \\
\hline Plantagineaceae & Scoparia dulcis L. & Ruma fada & VU B2ab(i,v);D2 & $\mathrm{NE}$ \\
\hline Poaceae & Dactyloctenium aegyptium (L.) Willd. & Guda gude & VU A2acd;D2 & $\mathrm{NE}$ \\
\hline Poaceae & Eleusine indica (L.) Gaertn. & Tuji & VU B1ab (iv,v);D2 & LC \\
\hline Polygalaceae & Securidaca longipedunculata Fresen. & Sanya & CR B1ab(i,ii,iv,v);D1 & NE \\
\hline Rhamnaceae & Ziziphus jujuba Mill. & Magarya & CRA2 c,d \&B2 b(v) & LC \\
\hline Rhamnaceae & Ziziphus spina-christi (L.) Desf. & Kurna & ENA2 d, B1 b(i) & NE \\
\hline Rubiaceae & Gardenia aqualla Stapf \& Hutch. & Gaude & CR B1ab (iv, v); D1 & $\mathrm{NE}$ \\
\hline Rutaceae & Citrus aurantiifolia (Christm.) Swingle & Lemun tsami & EN A2 d,e \&E & $\mathrm{NE}$ \\
\hline Sapotaceae & Vitellaria paradoxa C.F.Gaertn. & Kadanya & CRA3 c \&B1 b(i) & VU A1cd \\
\hline Solanaceae & Datura metel L. & Zakami & VU D2 & NE \\
\hline Vitaceae & Ampelocissus africana (Lour.) Merr. & Farun makiyaya & CR B12b (i,ii,iii,iv,v) & $\mathrm{NE}$ \\
\hline Vitaceae & Cissus populnea Guill \& Perr. & Loda & EN B2ab (iii,v) & $\mathrm{NE}$ \\
\hline Zygophyllaceae & Balanites aegyptiaca (L.) Delile & Aduwa & EN A1 b,c,d \&B1 c(i) & $\mathrm{NE}$ \\
\hline
\end{tabular}

Note: The table lists 108 taxa that are either EX or are threatened with extinction. One taxon qualifies as EX, 36 as CR, 25 as EN and 46 as VU (see also Figure 2).

EX, Extinct; NE, No entry; CR, Critically Endangered; EN, Endangered; VU, Vulnerable.

$(12.03 \%)$ each; impact of invasives $11(10.2 \%)$; urban residential development $8(7.40 \%)$; soil erosion $6(5.55 \%)$; grazing, habitat loss and timber with $5(4.6 \%)$ taxa each, respectively (Figure 4).

\section{Discussion}

One of the objectives of this study was to undertake an ethnobotanical survey of medicinal and economic plant diversity used in the treatment of various common ailments by the people of Katsina. The socio-demographic information showed that most of the respondents of the questionnaire dealing with ethnobotanical knowledge of the medicinal plants are males. This may not be unconnected to the fact that men are more engaged in outdoor activities such as farming, hunting and marketing and is also reflective of the religious and cultural setup of the people of northern Nigeria. Results also showed that most of the respondents lack formal education as already recorded by other studies around Katsina (e.g. Kankara et al. 2015; Mudansiru et al. 2016). This may affect negatively the traditional medicinal practices of knowledge sharing and dissemination, which prevents it from being lost. It was discovered during this study that most of the respondents were unwilling to share their ethnobotanical knowledge for fear of losing their inherited biological and genetic resources (see Etkin 2002). This has 


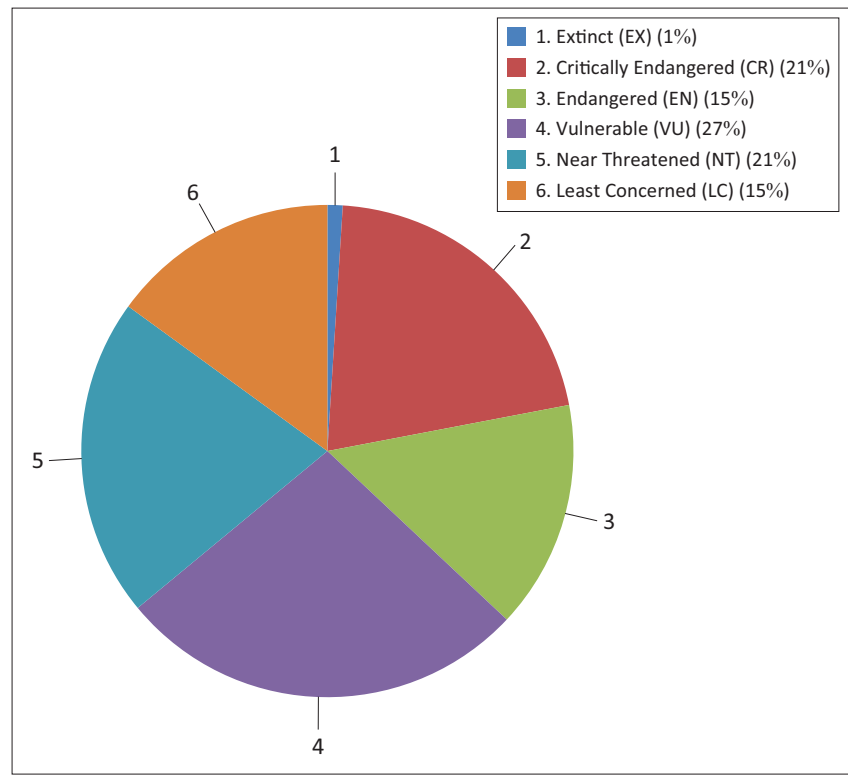

FIGURE 2: Percentage of threat category affecting the various medicinal and economic plants that occur in Katsina State, northwestern Nigeria.

elevated the need to embrace more proactive strides in the preservation and utilisation of this knowledge. However, the idea is not adequately understood by the local inhabitants as the majority of the older population who possess knowledge and information on the use and conservation of most of these medicinal plants are gradually declining in number without adequate documentation of their knowledge (Garba et al. 2014; Ekor 2013; Etkin 2002; Muul 1993).

Our ethnobotanical survey showed that Fabaceae has the highest record of 15 species of medicinal plants. This agrees with the findings of Kankara et al. (2015) in which the Fabaceae was recorded as the dominant family used in the treatment of various illnesses associated with maternal healthcare in Katsina State, Nigeria. This high occurrence of Fabaceae could be attributed to the fact that most of the species used for medicinal purposes belong to the Caesalpiniodeae and Mimosoideae clades, which are dominant in the tropical regions and therefore could survive the adverse effects of environmental changes in Savanna vegetation. Several other studies have also reported Fabaceae as the dominant family providing medicinal plants in Nigeria (Dambatta \& Aliyu 2011; El-Ghani 2016; Ene \& Atawodi 2012; Mudansiru et al. 2016).

A second objective of this study was to provide a conservation assessment of medicinal and economic plants found in the study area using the criteria and categories set up by the IUCN. Our results showed that more than half of the taxa studied (108) are threatened with extinction. This is alarming considering that most of the plants belonging to these groups are slow-growing native species that take several years before they reach maturity (Cunningham 1993). For example, while the cultivation of alternative sources of supply of popular, high conservation priority species has been proposed as one of the key solutions to the sustainable conservation management of the slow-growing or slow-reproducing species, commercial cultivation of such species is not an easy solution and at

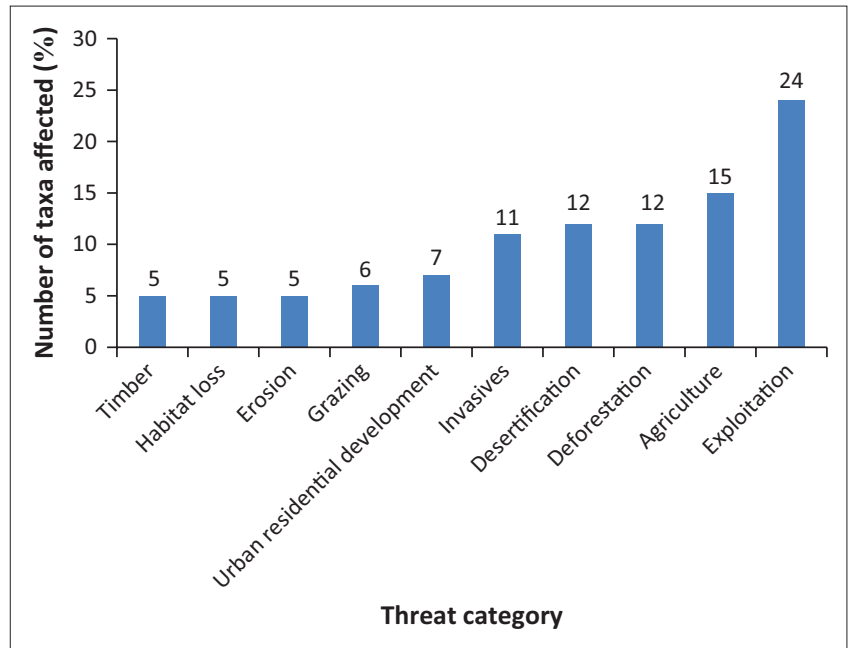

FIGURE 3: Various categories of threats faced by the plants and the number of taxa affected in each of the threat categories.

present is unlikely to be profitable because of the slow growth rates for most tree species and low prices paid for traditional medicines (Cunningham 1993). A good example of this limitation is the 60-year-old trial plot for Pterocarpus angolensis (Fabaceae) on the Mozambique coastal plain where growth on nutrient poor sands has been very slow (Cunningham 1993).

The Sudan Savanna vegetation is known to be characterised by the presence of sparsely scattered trees growing in patches. These trees (used as firewood) are by far the people's most important source of energy, accounting for more than $90 \%$ of the energy used in the region. It is known that the disappearance of trees dispossesses the land of its ability to retain water (FAO 2011). This may eventually lead to other detrimental effects such as drought, desertification leading to excessive erosion and the depletion of surface and groundwater (Smith 2000). With a loss of the natural vegetation, people are not only losing a valuable medicinal resource, but also the possibility of discovering new medicines (Aronson et al. 2010; Chukwuma et al. 2015). It has been reported that more than $80 \%$ of people in developing countries still rely on traditional medicines, which are largely sourced from plants (Etkin 2002; WHO 2001). By destroying these plants, rural people will lose access to their main supply of medicines. It is unfortunate that the level of understanding about these issues is at such a basic level in Nigeria, even at the very highest levels of government (Etkin 2002; Garba, Ajibade \& Appah 2014). Cunningham (1993) suggests that the conservation strategy for African medicinal plants must address the problem at two levels: recommendations which have socio-economic effects must be incorporated at the policy level and recommendations for conservation methodology must be addressed at the national and local levels. The recommendations cover the following areas: (1) international and national policy; (2) in-situ and ex-situ conservation methods; and (3) education and research.

Our results showed that out of the 169 taxa evaluated, only 12 had been evaluated globally. The majority of the plants were not recorded in the IUCN Red List database. 

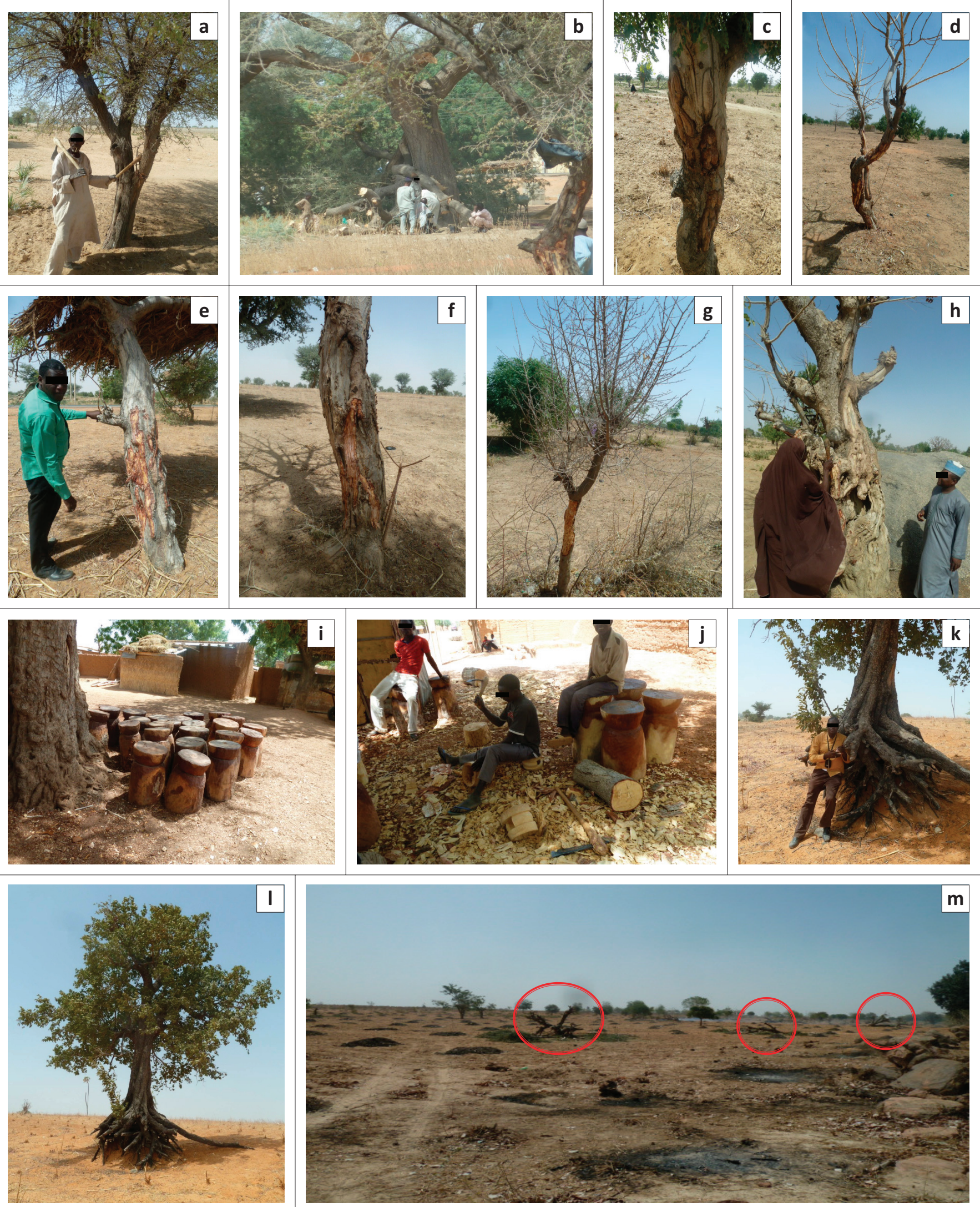

Source: Photos courtesy of A. Bello

FIGURE 4: Various categories of threat affecting the medicinal and economic plants in the Sudan Savanna vegetation zone: (a) Exploitation in Vachellia nilotica. (b) Exploitation in Faidherbia albida. (c) Exploitation in Sclerocarya birrea. (d) Exploitation in Lannea acida. (e) Exploitation in Anogeissus leiocarpa. (f) Exploitation in Annona senegalensis. (g) Exploitation in Entada africana. (h) Exploitation in Lannea acida. (i) Exploitation of Prosopis africana wood used for making mortar and pestle (j) and local stool making. (k-l) Roots of Diospyros mespiliformis exposed by erosion. (m) Exploitation and deforestation on Parkia biglobosa. (n) Neem (Azadirachta indica) invasion (o) competing with legume Bauhinia thonningii (twisting), ( $\mathrm{p}$ )-( $\mathrm{x}$ ) neem growing with legume Faidherbia albida and (y) neem growing with Annona senegalensis. (z), (aa), (bb) and (cc) Trunk of Diospyros mespiliformis used for timber making (dd) cattle grazing. 

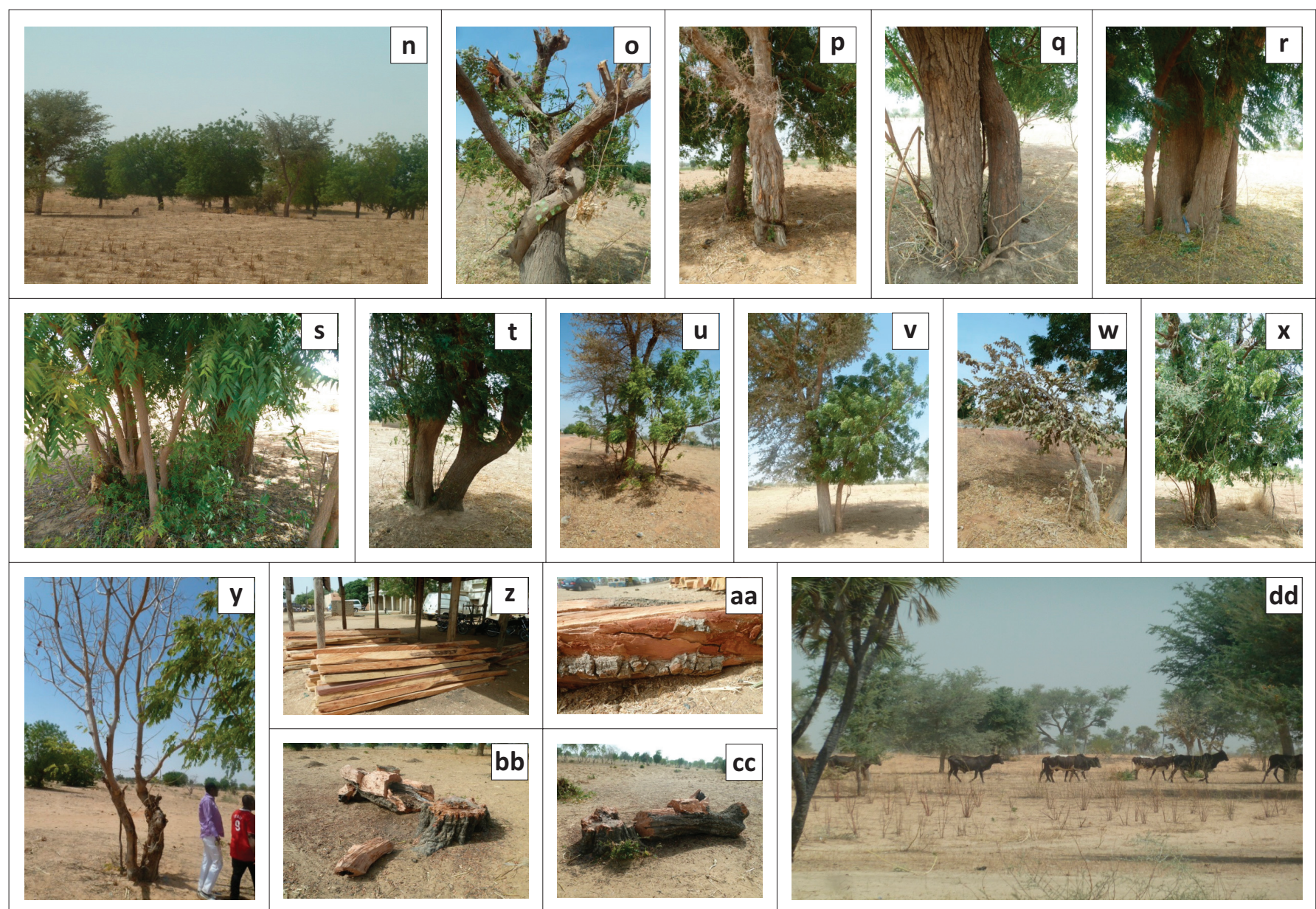

FIGURE 4 (Continues...): Various categories of threat affecting the medicinal and economic plants in the Sudan Savanna vegetation zone: (a) Exploitation in Vachellia nilotica. (b) Exploitation in Faidherbia albida. (c) Exploitation in Sclerocarya birrea. (d) Exploitation in Lannea acida. (e) Exploitation in Anogeissus leiocarpa. (f) Exploitation in Annona senegalensis. (g) Exploitation in Entada africana. (h) Exploitation in Lannea acida. (i) Exploitation of Prosopis africana wood used for making mortar and pestle (j) and local stool making. (k-l) Roots of Diospyros mespiliformis exposed by erosion. (m) Exploitation and deforestation on Parkia biglobosa. (n) Neem (Azadirachta indica) invasion (o) competing with legume Bauhinia thonningii (twisting), ( $\mathrm{p}$ )-( $\mathrm{x}$ ) neem growing with legume Faidherbia albida and (y) neem growing with Annona senegalensis. (z), (aa), (bb) and (cc) Trunk of Diospyros mespiliformis used for timber making (dd) cattle grazing.

This implies that the majority of species are either endemic to the Sudan Savanna vegetation zone or have never been evaluated. This vegetation type is usually neglected by the scientific community because of either a lack of abundant biodiversity or the harsh environmental conditions (Etkin 2002). The species are therefore prone to localised or global extinction stochastic effects. Some of the most important plant species observed were represented by only one individual throughout the region and include Prosopis africana listed as EN (Figures 5a-d) and Ficus ingens listed as CR (Figures $5 \mathrm{f}$ and $\mathrm{g}$ ). Prosopis africana is prized for its very strong red wood that is used for making mortar and pestle, as a charcoal used by the local blacksmiths, and for cooking. It is also used in making Islamic writing boards, chairs and other household accessories. It is being observed that the wood of $P$. africana is resistant to destruction by termites, which are known as major pests of woods. The only surviving individual of P. africana is found in the Baure Local Government Area although there is an unconfirmed report of its existence in the Kankia Local Government Area. The stem and the roots of $F$. ingens are known to be used for many ethnobotanical purposes. This might be the reason for the demise of most of the individuals. The only F. ingens individual found in Baure Local Government Area, estimated to have survived for about a century, has had its stem severely damaged and the roots have been dug out extensively (see Figures $5 \mathrm{f}$ and $\mathrm{g}$ ). Through this research, we are currently making some efforts to protect these remaining individuals through the adoption of the key sites of conservation concern and working with landowners to conserve the remaining threatened species.

We found one plant accessed as Extinct in this region. The plant Xeroderris stuhlmannii is a papilionoid legume in the family Fabaceae (Figure 5e). It is a deciduous tree of the Savanna woodland growing up to $12 \mathrm{~m}-15 \mathrm{~m}$ high. Its bark separates into thick scales and the trunk is known to exude blood-red sap when cut. Its white hysteranthous flowers are aggregated in grey- or rusty-tomentose panicles (Hutchinson \& Dalziel 1927). This plant is endemic to Savanna vegetation. The bark is purgative. It is used in traditional medicine to treat coughs, colds, rheumatic arthritis, stomach ache, dysentery, eye infections and wounds. Its roots are antihelminthic. When applied externally, a root decoction is very active against ringworm. A decoction of the bark is used as a treatment for internal parasites, elephantiasis, gonorrhoea, 

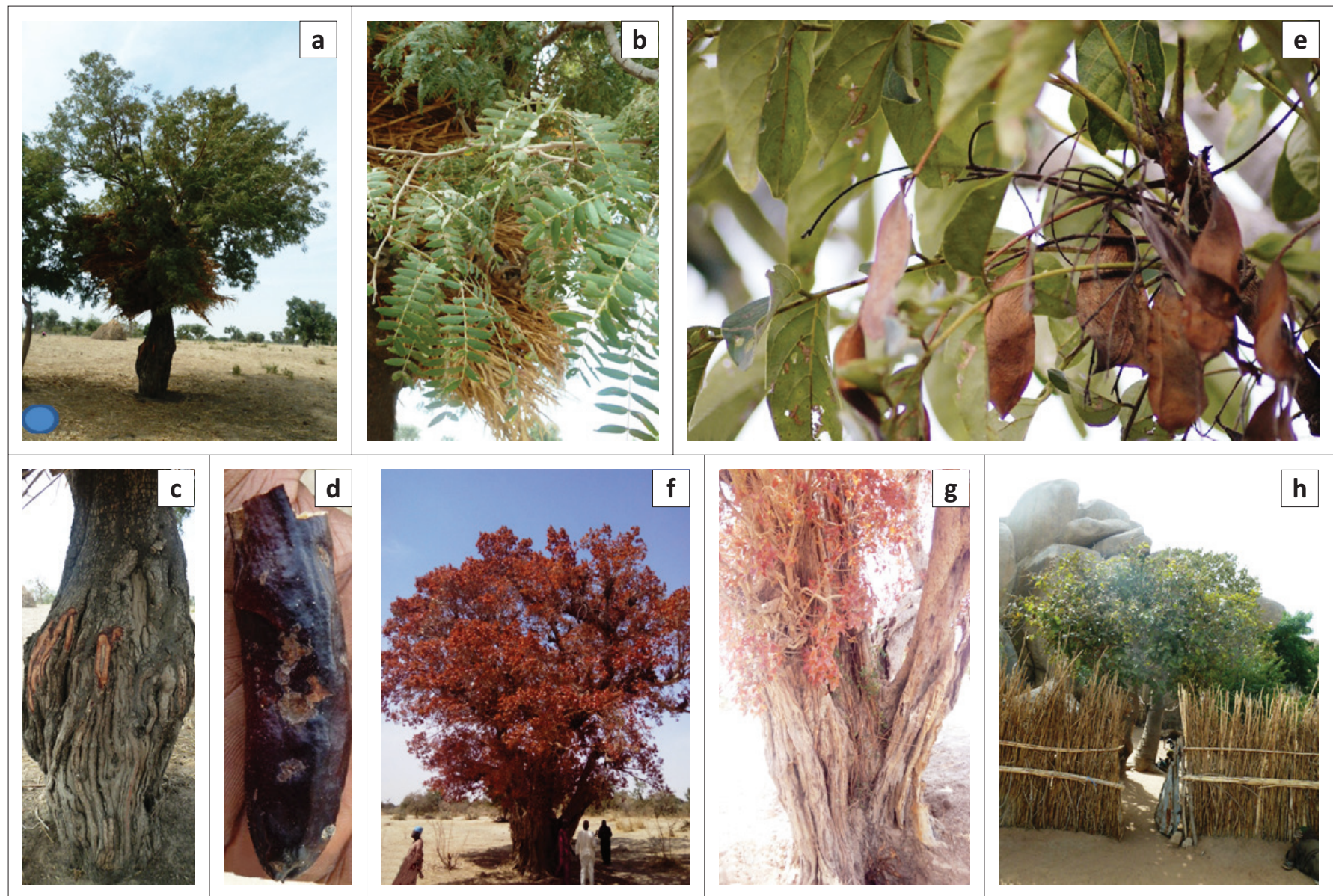

Source: Photos courtesy of A. Bello

FIGURE 5: Trees in which either none or only one last individual is found in the study area: (a) Prosopis africana tree. (b) Leaves of Prosopis africana. (c) Damaged stem of Prosopis africana. (d) Fruit of Prosopis africana. (e) Xeroderris stuhlmannii. (f) Ficus ingens. (g) Damaged stem bark of Ficus ingens. (h) Detarium microcarpum.

syphilis, dysmenorrhoea, chest pains and urinary complaints. Similarly, the pounded roots are applied to sores. This root usage might probably be a contributing factor to its disappearance because it is one of the slow-growing species. The leaves of X. stuhlmannii are used in the treatment of colds, coughs, wounds, stomach problems, amenorrhoea and malaria fever (Fern 2014).

This study found that the main reasons for the decline of these important medicinal and economic plants include overexploitation, agriculture, deforestation, desertification, alien invasives and the indiscriminate felling of trees for firewood, charcoal making and timber for construction and the making of utensils. These reasons have been reported several times as major threats to plants diversity and distribution (Allen et al. 2014; Dold \& Cocks 2002; Victor \& Dold 2003; Williams, Victor \& Crouch 2013). In Katsina, the most serious threat faced by plant taxa at risk of extinction now appears to be an overexploitation for medicinal purposes. Overexploitation has been a serious threat to medicinal plants for a long time. Plants mostly affected include mahogany (Khaya senegalensis (Desv.) A.Juss), African grape (Lannea acida A.Rich.), frankincense tree (Boswellia dalzielii Hutch.) and katsari (Albizia chevalieri Harms). There has been a significant decline in these species in particular. These plants are now being exploited commercially. This is a new potential threat, which has not yet received the attention it deserves. Possible solutions for overcoming the problem of overexploitation, apart from public education, are the establishment of $e x$-situ cultivation programmes to satisfy the commercial demand for these species and the enforcement of laws against indiscriminate felling of trees (Victor \& Dold 2003; Zizka et al. 2015). The new bill to regulate the trafficking of endangered species in Nigeria, which has been assented by the Nigerian President, is to bring the penalty provisions in line with economic realities and to act as a deterrent or deter people from trafficking and trading in endangered species. The act aims to discourage trafficking in endangered species and will encourage a culture of the preservation of endangered species.

Agriculture has recently created the most severe threat to species survival (Victor \& Dold 2003). This is not surprising because farmers in these communities often resort to burning large tracts of vegetation to clear land for crops. There is also a recent trend of felling trees for timber production. Trees mostly affected are the jackal berry (Diospyros mespiliformis Hochst. ex A.DC.; assessed as CR, see Figures 4z, 4aa, 4cc and $4 \mathrm{dd}$ ) and Faidherbia albida (Delile) A.Chev. (assessed as VU). These plants are slow growing and take a long time to achieve maturity. Unless appropriate measures are taken to conserve them, these plants will likely disappear before 2020 
because of the rate at which they are being exploited, the year set aside by the Convention on Biological Diversity (CBD) to prevent the extinction of the known threatened plants (CBD 2011).

Of more immediate concern is the threat posed by alien species in this region, particularly from the neem tree (Azadirachta indica A.Juss.) and eucalypts (Eucalyptus camaldulensis Dehnh.) (see Figure $4 n-x)$. A research study to check the menace of these alien species is currently underway by our research group. It has been observed in the field that neem tree grows attached to the roots of local plants, competing for nutrients and water. It could be that neem is stressing these native trees, leading to an earlier death. It has been reported that neem is allelopathic (Bello et al. 2010; Judd 2004), has a detrimental effect on many types of plants and is known to be a strong competitor. However, there is considerable empirical evidence that the neem tree has numerous positive medicinal benefits for local people elsewhere in West Africa and India and that if more information was made locally available as to their use and preparation, they could take off pressure of local medicinal species (Bello et al. 2010; Judd 2004). This will be considered a part of our further studies.

The information presented here underpins the importance and urgency for the Nigerian government and other conservation agencies to develop regionally appropriate conservation methods and strategies to minimise the main threats identified here and therefore reduce the risk of extinction of the threatened taxa identified. This aim is in accordance with the CBD's Target 12: 'By 2020, the extinction of known threatened species has been prevented and their conservation status, particularly of those most in decline, has been improved and sustained' (CBD 2010). This will ensure that the plants in the NT category are prevented from becoming threatened and will also enhance the securing of knowledge on data deficient taxa.

\section{Conclusion}

The results of this study have identified the most important economic and medicinal plants of the Sudan Savanna in Katsina State, northwestern Nigeria. The medicinal use of 43 plants species belonging to 20 different families was recorded. This showed that Savanna is naturally endowed with a diversity of important medicinal plants. It is critical therefore to promote their conservation and sustainable utilisation if they are not to disappear. We have also provided a conservation assessment of the plants based on the criteria developed by the IUCN. Most of the plants are already under threat and require conservation measures. Unfortunately, most of the people of the region seem to be unaware of the great threat facing medicinal plants in the wild as well as their own livelihoods. The data point to the critical need for better public education on these issues plus further research into conservation strategies and sustainable use of threatened plants and into better management of the useable exotic species identified in the region. The main current threats observed were because of human activities including overexploitation (for medicine), agriculture (other than livestock), deforestation, indiscriminate felling of trees for firewood and timber, and alien invasion.

Based on the findings of this study, our research supports and reiterates the following recommendations (see Gundu \& Adia 2014):

- The Nigerian government at all levels (federal, state and local) should consider implementing the two main methods of conserving biodiversity including ex-situ and in-situ conservation methods. Other methods of species conservation, such as natural regeneration and planting of indigenous plants, should be given priority through incentives and provision of nursery materials to rural people and resource users as they are adaptive and cost-effective.

- All sectors (governments and None Governmental Organisations [NGOs], as well as community-based initiatives) must ensure an integrated approach to tree multiplication and propagation of the local trees through policies and programmes and enlightenment campaigns. This is because universal education is key to biodiversity conservation.

- Researchers should undertake further research on Threatened and Near Threatened medicinal plants species and ensure the adequate identification and management of their critical habitats to inform conservation programmes and identify gaps in conservation actions.

- Threatened plants should be reassessed at least every five years and whenever new information becomes available.

- The government should enforce laws and regulations to protect endangered species. The plant part substitution method of plant conservation should be adopted, that is, the leaves and fruit of plant should be used instead of the root or bark, the use of which may lead to complete loss of the species.

\section{Acknowledgements}

This work was supported by funds from Nigeria Tertiary Education Trust Fund, Institution-Based Research, Umaru Musa Yar'adua University, Katsina, Nigeria (NTETF, IBR) to A. Bello. We thank Dr Tammy Elliot for editing the manuscript. We are also grateful to the respondents and all those who shared their bulk of knowledge and helped during the sampling and identification of the reported plants.

\section{Competing interests}

The authors declare that they have no financial or personal relationships that may have inappropriately influenced them in writing this article.

\section{Authors' contributions}

A.B. was the project leader. A.B., S.J., M.T.E., S.S.Y., S.S.K. and N.H.W. did the fieldwork. A.B., C.H.S. and M.M. analysed the data and wrote the manuscript. 


\section{References}

Allen, D., Bilz, M., Leaman, D.J., Miller, R.M., Timoshyna, A. \& Window, J., 2014, European Red List of medicinal plants, Publications Office of the European Union, Luxembourg.

Aronson, J., Blignaut, J.N., Milton, S.J., Le Maitre, D., Esler, K.J., Limouzin, A. et al., 2010 , 'Are socioeconomic benefits of restoration adequately quantified? A metaanalysis of recent papers (2000-2008) in restoration ecology and 12 othe scientific journals', Restoration Ecology 18(2), 143-154. https://doi.org/10.1111/ j.1526-100X.2009.00638.x

Hutchinson, J. \& Dalziel, J.M., 1927, 'Flora of West Tropical Africa, Vol. I., Part 2', Ostryoderris stuhlmannii (Taub.) Dunn ex Harms. JSTOR, Global plants, viewed 30 April 2017, from http://plants.jstor.org/stable/10.5555/al.ap.flora.fwta2945.

Fern, K., 2014, Xeroderris stuhlmannii, Useful Tropical Plants Database 2014, viewed 30 April 2017, from http://tropical.theferns.info/viewtropical.php?id=Xeroderris+ stuhlmannii

Bachman, S., Moat, J., Hill, A.V., De la Torre, J. \& Scott, B., 2011, 'Supporting Red List threat assessments with GeoCAT: Geospatial conservation assessment tool', Zookeys 150, 117-127. https://doi.org/10.3897/zookeys.150.2109

Bello, A., Aliero, A.A., Saidu, Y. \& Muhammad, S., 2011a, 'Phytochemical screening, polyphenolic content and alpha-glucosidase inhibitory potential of Leptadenic hastata (Pers.) decne', Nigerian Journal of Basic and Applied Science 19(2) 181-186.

Bello, A., Aliero, A.A., Saidu, Y. \& Muhammad, S., 2011b, 'Hypoglycaemic and hypolipidaemic effects of Leptadenia hastata (Pers.) decne in alloxan induced diabetic rats', Nigerian Journal of Basic and Applied Science 19(2), 187-192.

Bello, A., Khan, A.A., Umaru, A.M., Aliero, A.A. \& Shinkafi, B.Y., 2010, 'Effect of neem (Azadirachta indica) leaf litter on the growth of cowpea and millet', Katsina Journal of Natural and Applied Sciences 2(1), 152-155.

Borokini, T.I., 2014, 'A systematic compilation of endemic flora in Nigeria for conservation management', Journal of Threatened Taxa 6(11), 6406-6426. https://doi.org/10.11609/JoTT.04010.6406-26

CBD, n.d., Assessment of Biodiversity in Nigeria. Clearing house mechanism, viewed 22 February 2017, from http://www.chm-cbd.com.ng/index.php/en/biodiversity/ 22 February 2017, from http://www.chm-ch
assessment-of-the-threats-to-biodiversity

CBD, 2010, Conference of the Parties Decision X/2: Strategic plan for biodiversity 2011-2020, viewed 30 April 2017, from www.cbd.int/decision/cop/?id= 12268 .

CBD, 2015, Nigeria Fifth National Biodiversity Report, p. 89, viewed 30 April 2017, from https://www.cbd.int/doc/world/ng/ng-nr-05-en.pdf.

Chukwuma, E.C., Soladoye, M.O. \& Feyisola, R.T., 2015, 'Traditional medicine and the future of medicinal Plants in Nigeria', Journal of Medicinal Plants 3(4), 23-29.

Cunningham, A.B., 1993, African medicinal plants, United Nations Educational, Scientific and Cultural Organization, Paris, France.

Dambatta, S.H. \& Aliyu, B.S., 2011, 'A survey of major ethno medicinal plants of Kano north, Nigeria, their knowledge and uses by traditional healers', Bayero Journal of Pure and Applied Sciences 4(2), 28-34.

Dike, I.P. \& Obembe, O.O., 2012. 'Towards conservation of Nigerian medicinal plants', Journal of Medicinal Plants Research 6(19), 3517-3521. https://doi.org/10.5897/ JMPR10.612

Dold, A.P. \& Cocks, M.L., 2002. 'The trade in medicinal plants in the Eastern Cape Province, South Africa', South African Journal of Science 98(11-12), 589-597.

Ekor, M., 2013 'The growing use of herbal medicines: Issues relating to adverse reactions and challenges in monitoring safety', Frontiers in Pharmacology 4, 177.

El-Ghani, M.M.A., 2016, 'Traditional medicinal plants of Nigeria: An overview', Agriculture and Biology Journal of North America 7, 220-247.

Ene, A.C. \& Atawodi, S.E., 2012, 'Ethnomedicinal survey of plants used by the Kanuris of North-Eastern Nigeria', Indian Journal of Traditional Knowledge 11(4), 640-645.

Emma-Okafor, L.C. \& Ibeawuchi, I.I., 2010, 'Obiefuna Julius Chiedozie. Biodiversity Conservation for Sustainable Agriculture in Tropical Rainforest of Nigeria', New York Science Journal 3(1), 81-88.

Etkin, N.L., 2002, 'Local knowledge of biotic diversity and its conservation in rura Hausaland, Northern Nigeria 1', Economic Botany 56(1), 73-88. https://doi. org/10.1663/0013-0001(2002)056[0073:LKOBDA]2.0.CO;2

FAO, 2011, The state of the world's land and water resources for food and agriculture (SOLAW) - Managing systems at risk, Food and Agriculture Organization of the United Nations, Rome and Earthscan, London.
Garba, A.M., Ajibade, G.A. \& Appah, J., 2014, 'Backgrounds to the understanding of ethnobotanical practice among the Hausa people of Northern Nigeria', International Journal of Indigenous Medicinal Plants 47(1), 1539-1541.

Gbile, Z.O., 1992, 'Status of forest conservation for maintenance of biodiversity in Nigeria', in R.P. Adams \& J.E. Adams (eds.), Conservation of Plant Genes. DNA Banking and in vitro Biotechnology, Academic Press, San Diego, CA.

Gundu, E.D. \& Adia, J.E., 2014, 'Conservation methods of endangered species', Journal of Research in Forestry, Wildlife and Environmental 6(2), 76-83.

Hariramamurthi, G., 2000, 'The Genesis of the Medicinal Plants Conservation Network (MPCN)', Botanic Gardens Conservation International 1, 20, viewed 20 March 2017, from http://www.bgci.org/resources/article/0428/

Ihunweze, F., n.d., Dynamics of desertification: The case of Northern Nigeria, Online publication, viewed 25 March 2017, from https://www.academia.edu/6986631/ DYNAMICS_OF_DESERTIFICATION_THE_CASE_OF_NORTHERN_NIGERIA

IUCN, 2001, IUCN Red List categories and criteria version 3.1. IUCN species survival commission, IUCN Gland, Switzerland.

IUCN, 2012a, Guidelines for application of IUCN Red List criteria at regional and national levels v. 4.0, IUCN, Gland, Switzerland.

IUCN, 2012b, IUCN Red List categories and criteria v. 3.1, 2nd edn., IUCN, Gland, Switzerland.

Judd, M.P., 2004, 'Introduction and management of neem (Azadirachta indica) in smallholder's farm fields in the Baddibu Districts of The Gambia, West Africa', MSc thesis, Michigan Technological University, p. 111.

Kankara, S.S., Ibrahim, M.H., Mustafa, M. \& Go, R., 2015, 'Ethnobotanical survey of medicinal plants used for traditional maternal healthcare in Katsina State, Nigeria', South African Journal of Botany 97, 165-175. https://doi.org/10.1016/j sajb.2015.01.007

Keay, R.W.J., 1949, An outline of Nigerian vegetation, 3rd edn., Government Printer, Lagos.

Mudansiru, A., Haidara, A.M., Ibrahim, S., Zaharaddeen, U. \& Darma, A.M., 2016 'Ethno-botanical survey of some medicinal plants of Gumel Town, Jigawa State, Nigeria', Age 20(30), 5 .

Muul, I., 1993, Tropical forest integrated conservation strategies and concepts of critical mass MAB Digest No 15, UNSECO, Paris, France.

MedPlant, 2015, Conducting and communicating ethnobotanical research. A method manual, Global Diversity Foundation, p. 89, Canterbury, England.

Olofin, E.A., 1985, Human responses to the natural environment in the Kano region. Kano and its neighbours, ABU Press, Zaria.

Okigbo, R.N., Anuagasi, C.L. \& Amadi, J.E, 2009, 'Advances in selected medicinal plants indigenous to Africa', Journal of Medicinal Plants Research 3(2), 086-095.

Rodrigues, A.S.L., Pilgrim, J.D., Lamoreux, J.F., Hoffmann, M. \& Brooks, T.M., 2006, 'The value of the IUCN Red List for conservation', Trends in Ecology \& Evolution 21(2), 71-76. https://doi.org/10.1016/j.tree.2005.10.010

SANBI, 2010, Threatened species: A guide to Red Lists and their use in conservation, Threatened Species Programme, Pretoria, South Africa, $28 \mathrm{p}$.

Smith, D., 2000, 'When green earth turns into sand', NatGeo News, viewed December 2016, from Nationalgeographicnews.com

Taylor, D., 2015, The pharmaceutical industry and the future of drug development, $\mathrm{pp}$ 1-33, viewed 20 March 2017, from pubs.rsc.org; https://doi.org/10.1039/ 9781782622345-00001

Victor, J.E. \& Dold, A.P., 2003, 'Threatened plants of the Albany Centre of Floristic Endemism, South Africa', South African Journal of Science 99(9), 437-446.

Wakili, I., 2016, 'Buhari signs bill to stop endangered species trafficking', Daily Trust Newspaper, Media Trust, viewed 30 December 2016, from https://www. dailytrust.com.ng/news/general/buhari-signs-bill-to-stop-endangered-speciesdailytrust.com.ng/news/ger
trafficking/178410.html.

White, F., 1983, The vegetation of Africa. A descriptive memoir to accompany the UNESCO/AETFAT/UNSO vegetation map of Africa, Natural Resources Report XX, UNESCO, Paris.

Williams, V.L., Victor, J.E. \& Crouch, N.R., 2013, 'Red listed medicinal plants of South Africa: Status, trends, and assessment challenges', South African Journal of Botany 86, 23-35. https://doi.org/10.1016/j.sajb.2013.01.006

World Health Organization (WHO), 2001, Legal status of traditional medicine and complementary/alternative medicine: A worldwide review, World Health Organization, Geneva.

Zizka, A., Thiombiano, A., Dressler, S., Nacoulma, B.M.I., Ouédraogo, A., Ouédraogo, I., 2015, 'The vascular plant diversity of Burkina Faso (West Africa) - A quantitative analysis and implications for conservation', Candollea 70, 9-20. https://doi. org/10.15553/c2015v701a2 


\section{Appendix 1}

\section{Questionnaire for obtaining information on medicinal plants and their mode of administration.}

\section{Informants' consent for the participation in the study:}

I___ (name of informant) hereby give my full consent and conscious to participate in this study and declare that to the best of my knowledge, the information that I have provided are true, accurate and complete.

Date (Signature/Thumb impression of Informant)

\section{Informants' details:}

Name

Gender

Age

Occupation

Education

Location/Residence

\section{Data about medicinal plant and its use:}

Plant (Local name)

Habit (Tree/ Herb/ Shrub/Climber/

Plant part used

Cultivated/ Wild

If cultivated, cultivated for

If wild, availability in natural resources (easy/ difficulty/ very difficult)

Conservation needs

Conservation efforts made by Government and local residents

Method of collection and storage

Name of disease(s) treated

Method of crude drug preparation

Mode of administration

Dosage

Other uses (if any

\section{Remarks:}

Plant identified as (Botanical name and family)

Information provided by informants will be used for research purposes only 


\section{Appendix 2}

\section{Conservation data collection and assessment form}

\section{DEPARTMENT OF BIOLOGY, FACULTY OF NATURAL AND APPLIED SCIENCES, UMARU MUSA YARADUA UNIVERSITY, KATSINA, NIGERIA}

\section{APPENDIX 2}

SPECIES COLLECTION/ASSESSMENT FORM

Observer \& Locality (* Compulsory fields)

Collectior

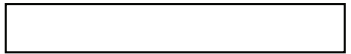

Description of locality

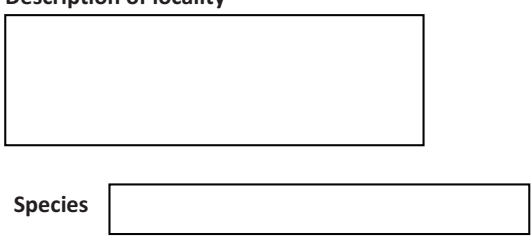

Dimensions of the population

Corner $1^{*}$

Corner $2^{*}$

Corner 3*

Corner $4^{*}$
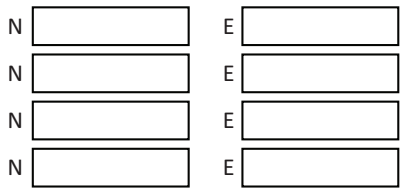

HABITAT

Landform:

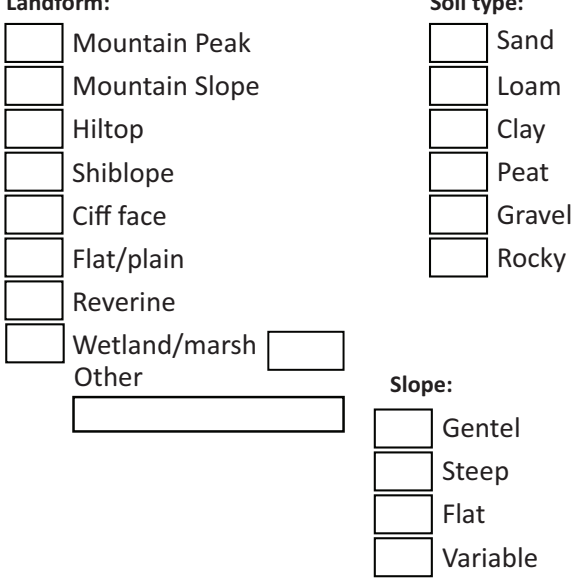

Land use:

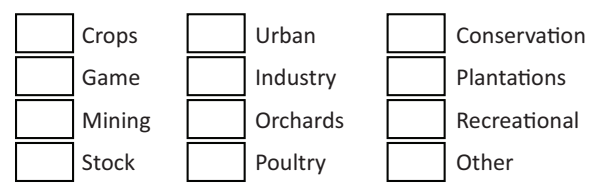

Note and Sketches

Rating - Degree of Impact

$$
\begin{array}{lll}
\text { 1. Very low } & \text { 2. Low } & \text { 3. medium } \\
\text { 4. High } & \text { 5. Very High } &
\end{array}
$$

Date

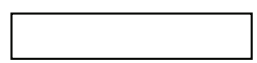

POPULATION INFORMATION (Compulsory fields)

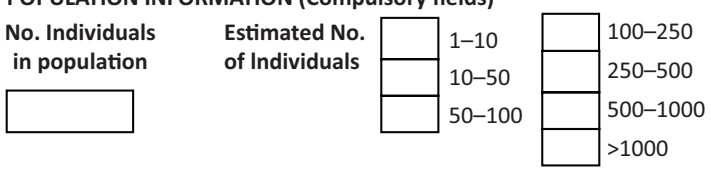

Distr. of plant

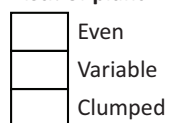

Area of extent

Dist.To next pop.

Population composition

$\square$ Adults $\square$ Juvenile $\square$ Seedlings $\square$ Dead Individuals
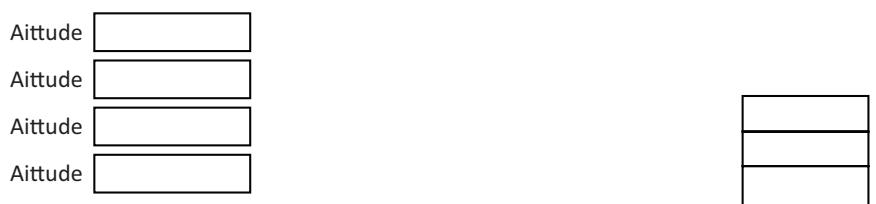

Geology THREATS \& DISTURBANCES*

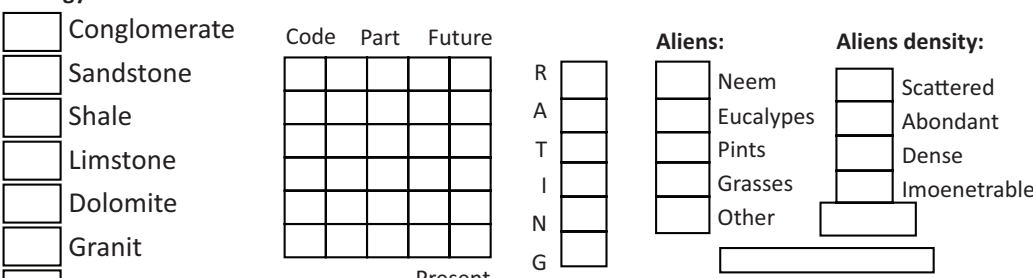

Present

G $\mathrm{km}$

Quarzite

Silcrete

Other

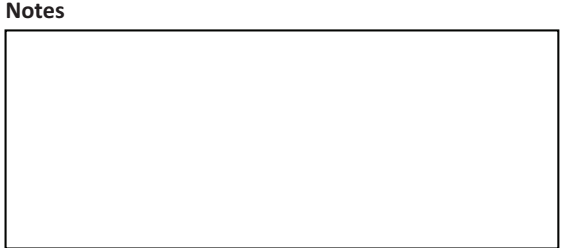

Aspect: $\mathrm{N}$

Ownership

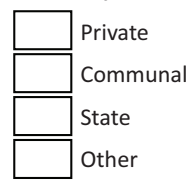

THREATA \& DISTURBANCES

1. Habitat loss degradation

A Agriculture

B Plantations

C Uvestock

D Abordonment

E Mining

F Timber harvesting

G Groundwater estractions

H Urbanization

I Roady/Railways

J Power Lines

K Dams

L Telecommunications

M Tourism/Recreation

$\mathrm{N}$ Aliens

O Too Frequent fines

P Too Irregular fines

Q Wrong season fines

2. Alens (Effect to Species)

A Competors

B Hybridusers 


\section{Appendix 3}

\section{International Union for the Conservation of Nature assessment categories and criteria.}

\section{SUMMARY OF THE FIVE CRITERIA (A-E) USED TO EVALUATE IF A TAXON BELONGS IN AN IUCN RED LIST THREATENED CATEGORY (CRITICALLY ENDANGERED, ENDANGERED OR VULNERABLE). ${ }^{1}$}

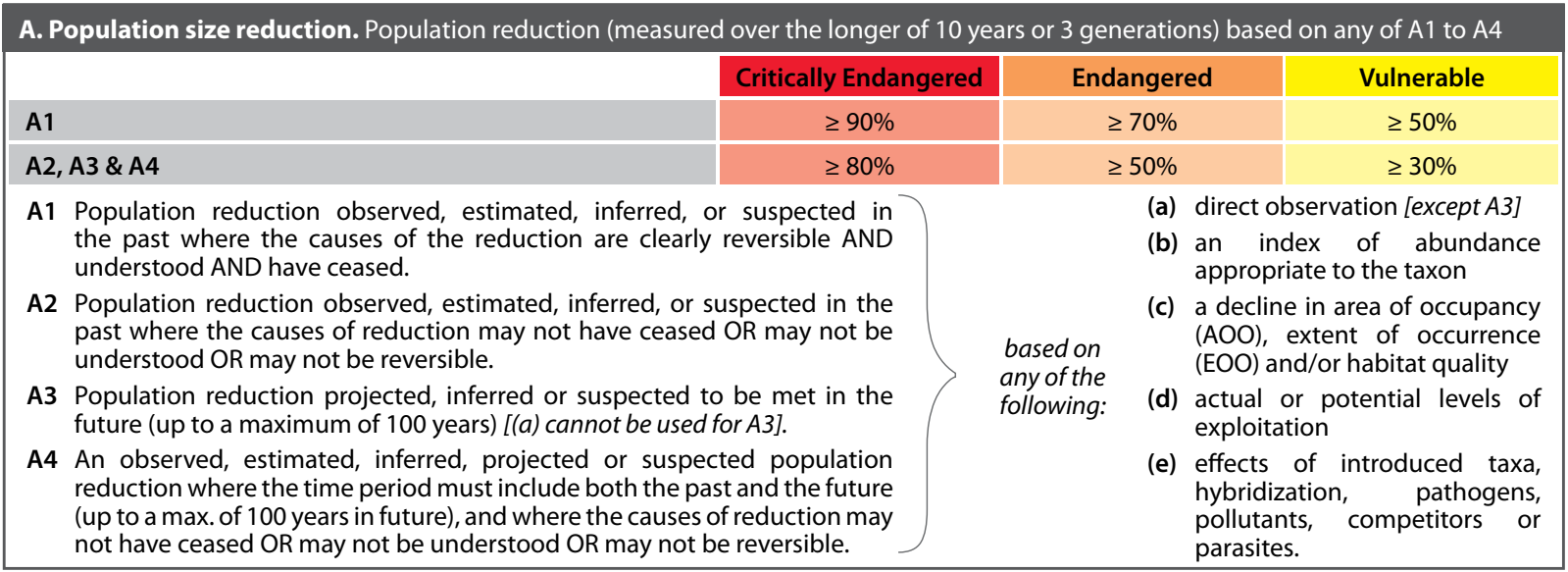

B. Geographic range in the form of either B1 (extent of occurrence) AND/OR B2 (area of occupancy)

\begin{tabular}{|l|c|c|c|}
\hline & Critically Endangered & Endangered \\
\hline B1. Extent of occurrence (EOO) & $<100 \mathrm{~km}^{2}$ & $<5,000 \mathrm{~km}^{2}$ \\
\hline B2. Area of occupancy (AOO) & $<10 \mathrm{~km}^{2}$ & $<20,000 \mathrm{~km}^{2}$ \\
\hline AND atnerable
\end{tabular}

AND at least 2 of the following 3 conditions:
(a) Severely fragmented OR Number of locations
$=1$
$\leq 5$
$\leq 10$
(b) Continuing decline observed, estimated, inferred or projected in any of: (i) extent of occurrence; (ii) area of occupancy; (iii) area, extent and/or quality of habitat; (iv) number of locations or subpopulations; (v) number of mature individuals
(c) Extreme fluctuations in any of: (i) extent of occurrence; (ii) area of occupancy; (iii) number of locations or subpopulations; (iv) number of mature individuals

\section{Small population size and decline}

\section{Number of mature individuals}

AND at least one of $\mathrm{C} 1$ or C2

C1. An observed, estimated or projected continuing decline of at least (up to a max. of 100 years in future):

C2. An observed, estimated, projected or inferred continuing decline AND at least 1 of the following 3 conditions:
(a) (i) Number of mature individuals in each subpopulation (ii) $\%$ of mature individuals in one subpopulation =
(b) Extreme fluctuations in the number of mature individuals

\begin{tabular}{|c|c|c|}
\hline Critically Endangered & Endangered & Vulnerable \\
\hline$<250$ & $<2,500$ & $<10,000$ \\
\hline $\begin{array}{c}25 \% \text { in } 3 \text { years or } \\
1 \text { generation } \\
\text { (whichever is longer) }\end{array}$ & $\begin{array}{c}20 \% \text { in } 5 \text { years or } \\
2 \text { generations } \\
\text { (whichever is longer) }\end{array}$ & $\begin{array}{c}10 \% \text { in } 10 \text { years or } \\
3 \text { generations } \\
\text { (whichever is longer) }\end{array}$ \\
\hline$\leq 50$ & $\leq 250$ & \\
\hline $90-100 \%$ & $95-100 \%$ & $\leq 1,000$ \\
\hline
\end{tabular}

\section{Very small or restricted population}

D. Number of mature individuals

\begin{tabular}{|c|}
\hline Critically Endangered \\
$<50$
\end{tabular}

Endangered
$<250$

\section{Vulnerable}

D2. Only applies to the VU category

Restricted area of occupancy or number of locations with

a plausible future threat that could drive the taxon to CR or EX in a very short time.

\section{E. Quantitative Analysis}

Indicating the probability of extinction in the wild to be:

\begin{tabular}{|c|c|c|}
\hline Critically Endangered & Endangered & Vulnerable \\
\hline \begin{tabular}{c|c}
$\geq 50 \%$ in 10 years or 3 \\
generations, whichever \\
is longer (100 years
\end{tabular} & $\begin{array}{c}\geq 20 \% \text { in } 20 \text { years or } 5 \\
\text { generations, whichever } \\
\text { is longer (100 years }\end{array}$ & $\geq 10 \%$ in 100 years \\
\hline & max.) & \\
\hline
\end{tabular}

D2. typically: $\mathrm{AOO}<20 \mathrm{~km}^{2}$ or number of locations $\leq 5$ max.) max.)

1 Use of this summary sheet requires full understanding of the IUCN Red List Categories and Criteria and Guidelines for Using the IUCN Red List Categories and Criteria. Please refer to both documents for explanations of terms and concepts used here. 